Document downloaded from:

http://hdl.handle.net/10251/81551

This paper must be cited as:

Gregori Verdú, S.; Tur Valiente, M.; Nadal, E.; Fuenmayor Fernández, FJ. (2016). Parametric model for the simulation of the railway catenary system static equilibrium problem. Finite Elements in Analysis and Design. 115:21-32. doi:10.1016/j.finel.2016.02.007.

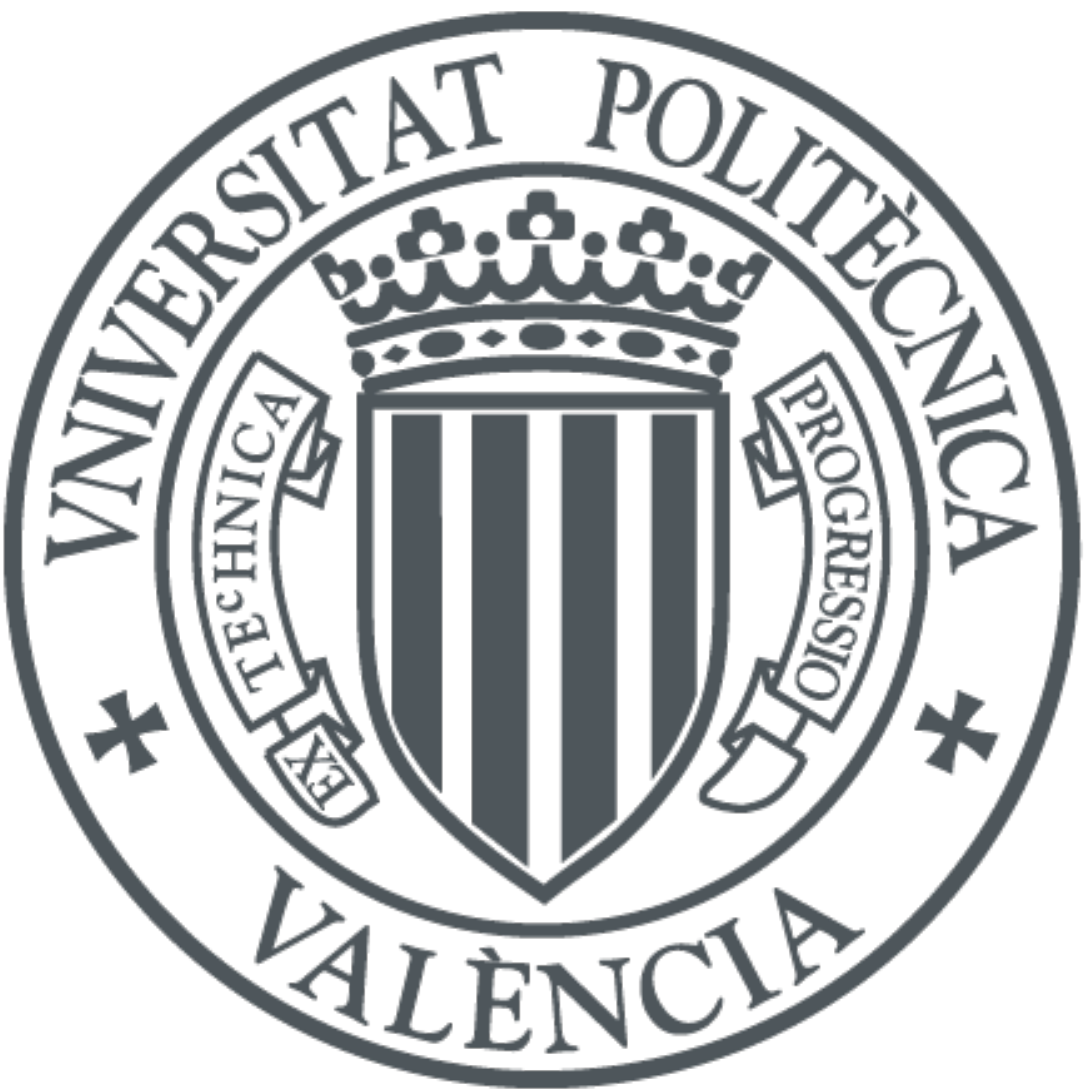

The final publication is available at

http://dx.doi.org/10.1016/j.finel.2016.02.007

Copyright Elsevier

Additional Information 


\title{
Parametric model for the simulation of the railway catenary system static equilibrium problem
}

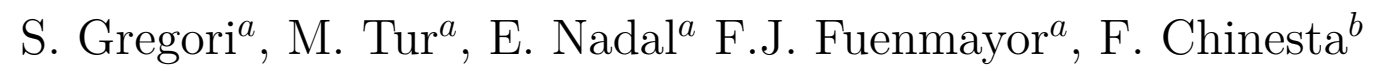 \\ ${ }^{a}$ Centro de Investigación de Tecnología de Vehículos, \\ Departamento de Ingeniería Mecánica y de Materiales, Universitat Politècnica de València, \\ Camino de Vera s/n, 46022 Valencia, Spain \\ ${ }^{b}$ UMR CNRS-Centrale Nantes, 1 rue de la Noe, BP 92101, F-44321 Nantes Cedex 3, France
}

Julio, 2015

\begin{abstract}
Dynamic simulations of pantograph-catenary interaction are nowadays essential for improving the performance of railway locomotives, by achieving better current collection at higher speeds and lower wear of the collecting parts. The first step in performing these simulations is to compute the static equilibrium of the overhead line. The initial dropper lengths play an important role in hanging the contact wire at an appropriate height. From a classical point of view, if one wants to obtain the static equilibrium configuration of the system for different combinations of dropper lengths, one static problem must be solved for each combination of lengths, which involves a prohibitive computational cost. In this paper we propose a parametric model of the catenary, including the undeformed dropper lengths as extracoordinates of the problem. This multidimensional problem is efficiently solved by means of the Proper Generalized Decomposition (PGD) technique, avoiding the curse of dimensionality issue. The capabilities and performance of the proposed method are shown by numerical examples.
\end{abstract}




\section{Introduction}

The overhead line equipment, or the so called overhead catenary, is the system responsible for providing electric energy to railway locomotives by means of a pantograph. As a result of the pantograph-contact wire interaction and the dynamic response of the system, a contact force is generated which varies in time. The overhead line is designed to operate at the smallest possible contact force (to minimize wear due to friction) but maintaining at least a minimum value to ensure that the pantograph always remains in contact with the wire. The numerical models used to simulate this system are a useful tool in catenary design for achieving better high-speed current collection [1, 2, 3]. Fig. 1 shows the main elements of a typical railway catenary.

Many factors influence the dynamics of the overhead system and affect the contact force, these include dropper lengths, which are parameters that can be easily modified in engineering practice using the current catenary-stringing technology. The static position of the contact wire largely depends on the length and position of the droppers. Thus, the interaction of the pantograph with the contact wire and the contact force generated depends on the dropper lengths. In fact, some amount of the so-called presag of the contact wire (deviations of the contact wire height from the horizontal position) has been shown to improve catenary performance at high-speeds $[4,5]$. It is still an open question whether or not there is an optimal droppers length for a certain pantograph and train speed that provides the best performance in terms of contact force. Numerical simulation tools can help in solving this issue. However, at the present time, the analysis of the influence of undeformed dropper lengths on the dynamics of the system would require a great number of simulations for different combinations of these parameters, which would be unfeasible in practice with traditional finite element technology.

The aim of this paper is to present a numerical method able to perform this type of analysis at a reasonable computational cost. In particular, there is an especial interest in finding the static equilibrium position of the railway catenary system for any combination of dropper lengths. By using the Proper Generalized Decomposition (PGD) technique it is possible to solve parametric models that are defined in high dimensional spaces, such as in the problem at hand, in which undeformed dropper lengths are introduced as extra-coordinates. 
PGD [6] is a Model Order Reduction (MOR) technique which can easily solve multidimensional problems. PGD has already successfully addressed a variety of problems, including shell-type geometries [7, 8], shape optimization problems [9], computational rheology [10], linear elastic fracture mechanics [11] or mechanic simulation for biological tissues $[12,13]$ among others, in a multidimensional framework. Space-time decompositions are dealt with in [14] under a PGD approach. The errors of the PGD solutions are studied in [15]. PGD is thus able to provide a multiparametric solution of the problem that explicitly depends on the parameters to be indentified (in this case dropper lengths) and avoids the curse of dimensionality issue when a large number of parameters are considered. The interested reader is addressed to [16] and the references therein for a deeper analysis of this aspect.

The main interest of the proposed method is to obtain a solution of the static equilibrium position, required to simulate the dynamic interaction, for any combination of dropper lengths. With the parametric solution it is possible to perform an efficient geometry optimization process of the catenary, based on different criteria, such as the minimal standard deviation of the contact force. With a parametric dynamic solution of the problem, the effect of wrong stringing, which leads to a static configuration other than the one designed, can be reproduced and analyzed.

The paper is organized as follows. The overhead line and the elements which compose the catenary are described in Section 2. In Section 3 the finite element model of the catenary is introduced. This model is based on the absolute nodal coordinate formulation (ANCF). On the basis of the virtual work principle, in Section 4 the static equilibrium problem is presented from a classical point of view. In Section 5 the static equilibrium problem is dealt with the PGD approach. The proposed formulation is given in two versions: i) without considering dropper slackening and ii) including the effect of dropper slackening. A linearized problem is also presented in order to reduce the computational cost. The accuracy and performance of the method is analized in Section 6 through some numerical examples. Finally, the conclusions are summarized in Section 7. 


\section{Description of the overhead line}

Fig. 1 shows a high-speed train catenary. The catenary is mainly composed of two groups of components, structural elements and cables. Masts, brackets and registration arms are responsible for supporting the entire cabling in the desired position. The cables include the messenger or carrier wire, droppers and contact wire. The messenger wire hangs from the brackets at regular intervals. Its main aim is to hold the contact wire at the required height from the track. This can be achieved by means of droppers clamped to the messenger and contact wire at certain points in every span. The contact wire transmits electrical power to the locomotive through the pantograph head on the locomotive roof. Some types of catenaries include stitch wires near the masts in order to reduce the variation of the stiffness along the span. Both the messenger and contact wires are prestressed and keep the tension constant with the aid of a compensation system located at both ends of each section along the overhead line.

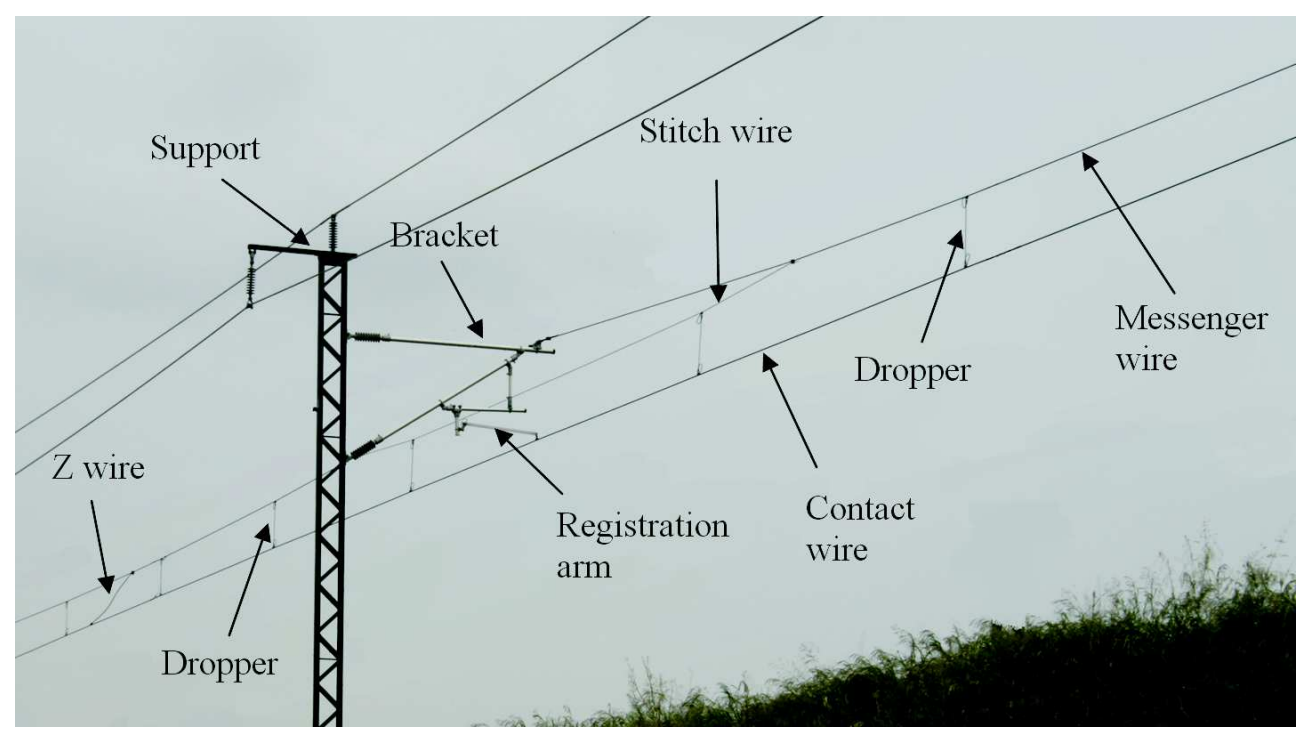

Figure 1: Photo of a high-speed train catenary.

Viewed from above, the catenary follows a zigzag pattern from one bracket to another. This stagger is designed to guarantee uniform wear on the contact strip of the pantograph collectors. Another important geometric issue in many catenaries is their presag, which reduces the variations in contact force caused by the reduced stiffness in the central region of the spans, and is controlled by means of appropriate dropper lengths. 
It is important to point out that small changes in certain parameters, such as the undeformed dropper lengths, may change the height of the contact wire and therefore affect interaction with the pantograph. Also, if the initial length of a dropper is larger than a certain value, the dropper can slacken and fail to hold the contact wire in the static position.

\section{Catenary finite element model}

The catenary system was modeled by finite elements. Only the main features of the model are summarized here (for further information see [17]). An example of this model is depicted in Fig. 2, in which the nodes are plotted as circles. A beam element based on the absolute nodal coordinate formulation (ANCF) is employed to model the cables. The original 3D ANCF element was proposed in $[18,19]$ and used for railway catenary models in $[20,21]$. For the interested reader, a good comparison between this element and the elements based on the classical formulation can be found in [22].

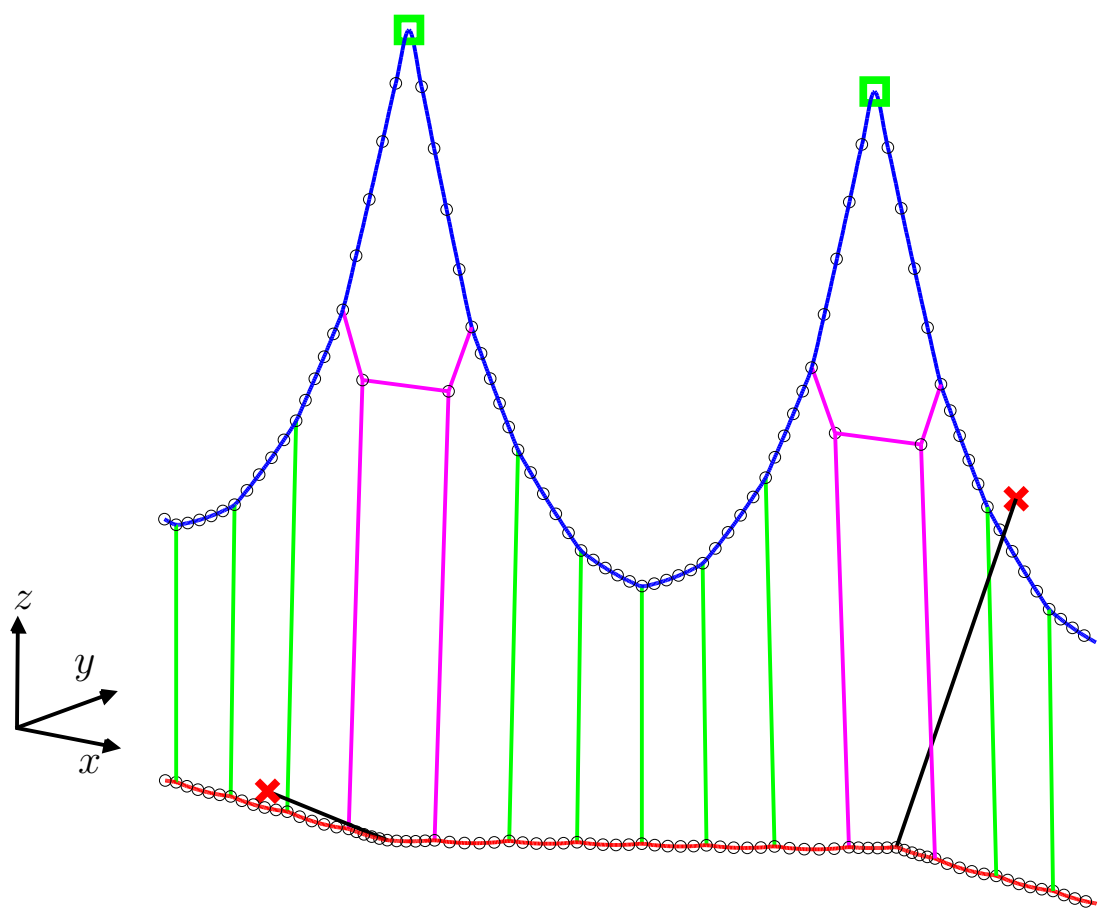

Figure 2: Finite element model of the catenary. 
Catenary wires are much longer than their cross-sectional area, so that the torsional effects can be neglected. This results in the element introduced in [23] with only 6 degrees of freedom per node, taking into account axial and bending deformations. In this paper, this type of element is called a 'cable element' and is used to model both the messenger and the contact wires. Droppers and registration arms are modeled as a single large displacement nonlinear element called a 'bar element' throughout the paper. The bar element is only capable of transmitting axial forces in traction and slackens under compressive forces.
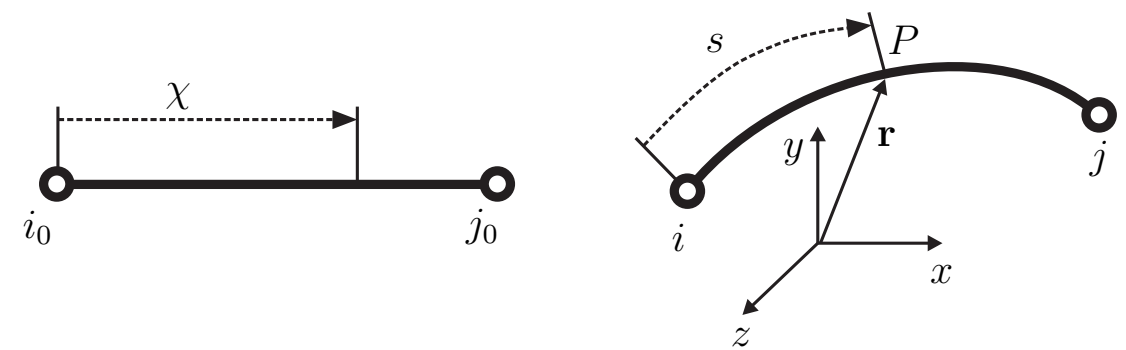

Figure 3: Undeformed and deformed configurations of the ANCF element.

In this model the masts and brackets are replaced by suitable boundary conditions. Dirichlet boundary conditions are applied at the ends of the registration arms joined to the brackets (nodes marked with a cross in Fig. 2). Spring-damping elements are used to simulate the supports (nodes marked with a square in Fig. 2).

Fig. 3 shows a scheme of the reference and deformed configurations of an ANCF cable element. The vector of degrees of freedom of a cable element of nodes $i$ and $j$ containing the coordinates and their gradient is:

$$
\mathbf{q}_{c}=\left[\begin{array}{llllllllllll}
x_{i} & y_{i} & z_{i} & \frac{\partial x_{i}}{\partial \chi} & \frac{\partial y_{i}}{\partial \chi} & \frac{\partial z_{i}}{\partial \chi} & x_{j} & y_{j} & z_{j} & \frac{\partial x_{j}}{\partial \chi} & \frac{\partial y_{j}}{\partial \chi} & \frac{\partial z_{j}}{\partial \chi}
\end{array}\right]^{T}
$$

where $\chi \in\left[0, l_{0}\right]$ is the local coordinate, $l_{0}$ being the length of the undeformed element. In a deformed configuration, the absolute position coordinates $\mathbf{r}(\chi)$ are defined by means of a cubic polynomial that can be written as:

$$
\mathbf{r}(\chi)=\mathbf{S}_{c}(\chi) \mathbf{q}_{c}
$$


The interpolation is defined as:

$$
\begin{aligned}
\mathbf{S}_{c}(\chi)= & {\left[\begin{array}{cccccccccccc}
S_{c 1} & 0 & 0 & S_{c 2} & 0 & 0 & S_{c 3} & 0 & 0 & S_{c 4} & 0 & 0 \\
0 & S_{c 1} & 0 & 0 & S_{c 2} & 0 & 0 & S_{c 3} & 0 & 0 & S_{c 4} & 0 \\
0 & 0 & S_{c 1} & 0 & 0 & S_{c 2} & 0 & 0 & S_{c 3} & 0 & 0 & S_{c 4}
\end{array}\right] } \\
& S_{c 1}(\xi)=1-3 \xi^{2}+2 \xi^{3} \\
& S_{c 3}(\xi)=3 \xi^{2}-2 \xi^{3}(\xi)=l_{0}\left(\xi-2 \xi^{2}+\xi^{3}\right)
\end{aligned}
$$

The coordinate $\xi=\chi / l_{0} \in[0,1]$ denotes the normalized local coordinate. These Hermite cubic polynomials guarantee the $C^{1}$ continuity between elements.

The element vector of degrees of freedom of the bar element contains only the absolute positions of the two nodes as:

$$
\mathbf{q}_{b}=\left[\begin{array}{llllll}
x_{i} & y_{i} & z_{i} & x_{j} & y_{j} & z_{j}
\end{array}\right]^{T}
$$

The interpolation used for this element is linear, and the length of the undeformed element does not appear explicitly in the shape functions as shown in:

$$
\begin{aligned}
& \mathbf{S}_{b}(\chi)=\left[\begin{array}{cccccc}
S_{b 1} & 0 & 0 & S_{b 2} & 0 & 0 \\
0 & S_{b 1} & 0 & 0 & S_{b 2} & 0 \\
0 & 0 & S_{b 1} & 0 & 0 & S_{b 2}
\end{array}\right] \\
& S_{b 1}(\xi)=-\frac{\xi-1}{2} \quad S_{b 2}(\xi)=\frac{\xi+1}{2}
\end{aligned}
$$

For the sake of clarity, in the following sections the equations are particularized for cable elements, since they are directly applicable to bar elements only by neglecting the term of bending deformations. Subscripts $c$ and $b$ are deleted for simplicity in the notation.

\section{Static equilibrium problem}

This section introduces the classical FEM formulation to solve the static equilibrium configuration of a railway catenary under gravitational effects. In a catenary system, before computing the static equilibrium position, the so-called 'shape-finding' or initial configuration problem must be solved. The goal of this problem is to compute the undeformed 
lengths of the elements fulfilling the force equilibrium equations and all the constraints introduced during the catenary stringing. The tension in the messenger and the contact wire, and the height of the latter are the main constraints taken into account. A thoroughly detailed account of a method of dealing with the 'shape-finding' problem of the overhead line can be found in [17]. In this contribution, it is assumed that the initial configuration problem is solved, i.e. the undeformed length of all the cables is given. The static equilibrium position under gravitational forces is sought for possibly different values of the undeformed dropper lengths.

The formulation of the problem is obtained by using the virtual work principle. Let $\Omega$ be defined as the spatial domain of a certain railway catenary system, i.e. the catenary components modeled by FE. If $\Omega$ is discretized into $N_{e}$ elements such that $\Omega=\cup_{i}^{N_{e}} \Omega_{i}^{e}$ and $\Omega_{i}^{e} \cap \Omega_{j}^{e}=\emptyset, i \neq j$, the total virtual work of internal forces obtained as the combination of each element is

$$
\delta W_{i n t}=\sum_{e=1}^{N_{e}} \delta W_{i n t}^{e}
$$

For an element, this work is due to the contribution of the axial and the bending strains,

$$
\delta W_{i n t}^{e}=\int_{\Omega^{e}}\left(E A \delta \varepsilon_{L} \varepsilon_{L}+E I \delta \kappa \kappa\right) d \chi
$$

where $E$ represents the Young's modulus, $A$ denotes the cross-sectional area, $I$ is the second moment of area and, $\varepsilon_{L}$ and $\kappa$ represent the axial deformation and the curvature of the element, respectively. The axial strain can be defined using the Green strain tensor as:

$$
\varepsilon_{L}=\frac{1}{2}\left(\frac{d \mathbf{r}}{d \chi} \cdot \frac{d \mathbf{r}}{d \chi}-1\right)
$$

From the Frenet-Serret frame, the curvature [24] is defined as:

$$
\kappa=\left|\frac{d^{2} \mathbf{r}}{d s^{2}}\right|=\frac{\left|\frac{d \mathbf{r}}{d \chi} \times \frac{d^{2} \mathbf{r}}{d \chi^{2}}\right|}{\left|\frac{d \mathbf{r}}{d \chi}\right|^{3}}
$$

where $s$ is the local coordinate in the deformed configuration as showed in Fig. 3. Since on the catenary wires the axial strains are observed to be small, $d s \approx d \chi$ and the definition 
of curvature can be approximated by [19]:

$$
\kappa \approx\left|\frac{d^{2} \mathbf{r}}{d \chi^{2}}\right|
$$

Computing the variations of the axial deformation and the curvature and replacing them into (7), the virtual work of internal forces results in:

$$
\delta W_{i n t}^{e}=\int_{\Omega^{e}}\left[E I \frac{d^{2} \delta \mathbf{r}}{d \chi^{2}} \cdot \frac{d^{2} \mathbf{r}}{d \chi^{2}}+\frac{E A}{2} \frac{d \delta \mathbf{r}}{d \chi} \cdot \frac{d \mathbf{r}}{d \chi}\left(\frac{d \mathbf{r}}{d \chi} \cdot \frac{d \mathbf{r}}{d \chi}-1\right)\right] d \chi
$$

On the other hand, the force of gravity acts on the catenary. For an element, the virtual work caused by this external force is:

$$
\delta W_{e x t}^{e}=\int_{\Omega^{e}} \delta \mathbf{r} \cdot \mathbf{g} d \chi
$$

where $\mathbf{g}=\left\{\begin{array}{lll}0 & 0 & -g A \rho\end{array}\right\}^{T}, g$ is the gravitational constant and $\rho$ is the density. Finally, the weak form of the static problem is obtained by equating (11) and (12) and accounting for all the element contributions. It consists of finding $\mathbf{r}(\chi)$ for all the admissible $\delta \mathbf{r}$, such that:

$$
\sum_{e=1}^{N_{e}} \int_{\Omega^{e}}\left[E I \frac{d^{2} \delta \mathbf{r}}{d \chi^{2}} \cdot \frac{d^{2} \mathbf{r}}{d \chi^{2}}+\frac{E A}{2} \frac{d \delta \mathbf{r}}{d \chi} \cdot \frac{d \mathbf{r}}{d \chi}\left(\frac{d \mathbf{r}}{d \chi} \cdot \frac{d \mathbf{r}}{d \chi}-1\right)-\delta \mathbf{r} \cdot \mathbf{g}\right] d \chi=0, \quad \forall \delta \mathbf{r}
$$

After the assembly process taking into account all the Dirichlet boundary conditions, a nonlinear algebraic system of equations is obtained. The static equilibrium equation for the whole catenary system reads:

$$
\mathbf{f}(\mathbf{q})=\mathbf{0}
$$

which can be solved using for example the Newton-Raphson method. Note that if one wish to obtain the static equilibrium position for different sets of initial dropper lengths, the domain $\Omega$ changes, therefore a new static problem (Eq. (13)) must be solved. 


\section{Proper Generalized Decomposition approach}

The objective of this section is to solve the static equilibrium problem (13) for any initial configuration of dropper lengths. For this purpose the PGD model order reduction technique is used. In this approach the geometrical parameters 'dropper lengths' are considered as extra-coordinates of the problem. Thus, the unknown field of absolute positions, and also its variations, now depends on both the spatial and dropper length coordinates, $\mathbf{r}=\mathbf{r}\left(\chi, l_{p 1}, l_{p 2}, \ldots\right)$. For the sake of simplicity, in this section the formulation that includes only one undeformed dropper length as extra-coordinate of the static equilibrium problem is first presented, therefore $\mathbf{r}\left(\chi, l_{p}\right)$. In the last part of the present section, the proposed formulation is generalized to $\mathscr{N}$ undeformed dropper lengths extra-coordinates.

Remark: Note the difference between $l_{0}$, the constant value of the undeformed length of an element, and $l_{p}$, which represents any possible value of the undeformed dropper length, taking values in an appropriate interval defined later.

\subsection{PGD formulation}

With the addition of one dropper length as extra-coordinate $l_{p}, l_{p} \in \Omega_{l}$, its domain of variation $\Omega_{l}=\left[l_{p}^{-}, l_{p}^{+}\right]$is defined as the values of the lengths for which it is desired to obtain the static equilibrium. The variational problem (13) extends to the whole geometry of the catenary, $\Omega$ and to the domain $\Omega_{l}$. The new problem consists of finding the absolute position $\mathbf{r}\left(\chi, l_{p}\right)$ such that for all virtual displacement $\delta \mathbf{r}$ :

$$
\sum_{e=1}^{N_{e}} \int_{\Omega_{l}} \int_{\Omega^{e}}\left[E I \frac{d^{2} \delta \mathbf{r}}{d \chi^{2}} \cdot \frac{d^{2} \mathbf{r}}{d \chi^{2}}+\frac{E A}{2} \frac{d \delta \mathbf{r}}{d \chi} \cdot \frac{d \mathbf{r}}{d \chi}\left(\frac{d \mathbf{r}}{d \chi} \cdot \frac{d \mathbf{r}}{d \chi}-1\right)-\delta \mathbf{r} \cdot \mathbf{g}\right] d \chi d l_{p}=0, \quad \forall \delta \mathbf{r}
$$

Note that the spatial domain $\Omega$ depends on the initial length of the dropper $l_{p}$, which is problematic for the proposed formulation. In the same way as in [9], the following change of variable is applied to (15) in order to circumvent this problem:

$$
\chi=l_{p} \tilde{\chi}, \quad \tilde{\chi} \in[0,1]
$$


With the introduced change of variables, the contribution of an element to the static equilibrium reads:

$$
\int_{\Omega_{l}} \int_{0}^{1}\left[\frac{E I}{l_{p}^{2}} \frac{d^{2} \delta \mathbf{r}}{d \tilde{\chi}^{2}} \cdot \frac{d^{2} \mathbf{r}}{d \tilde{\chi}^{2}}+\frac{E A}{2 l_{p}^{2}} \frac{d \delta \mathbf{r}}{d \tilde{\chi}} \cdot \frac{d \mathbf{r}}{d \tilde{\chi}}\left(\frac{1}{l_{p}^{2}} \frac{d \mathbf{r}}{d \tilde{\chi}} \cdot \frac{d \mathbf{r}}{d \tilde{\chi}}-1\right)-\delta \mathbf{r} \cdot \mathbf{g}\right] l_{p} d \tilde{\chi} d l_{p}
$$

Now it is possible to solve this problem with the PGD technique. PGD is based on a separate representation of the unknown field. In this case:

$$
\mathbf{r}\left(\tilde{\chi}, l_{p}\right) \approx \mathbf{r}^{n}=\sum_{i=1}^{n} \mathbf{R}_{i}(\tilde{\chi}) L_{i}\left(l_{p}\right)
$$

where each mode is composed of an $\mathbf{R}_{i}(\tilde{\chi})$ function that only depends on the spatial coordinate $\tilde{\chi}$, and an $L_{i}\left(l_{p}\right)$ function that depends on the extra-coordinate $l_{p}$.

In order to account for the non-homogeneous Dirichlet boundary conditions present in this problem (the absolute position of the points that connect the wires with the fixed structure), let us consider a function $\mathbf{g}\left(\tilde{\chi}, l_{p}\right)$ that satisfies these conditions. It is possible to state $\mathbf{g}\left(\tilde{\chi}, l_{p}\right)$ in a separate form

$$
\mathbf{g}\left(\tilde{\chi}, l_{p}\right)=\mathbf{G}_{\chi}(\tilde{\chi}) G_{l}\left(l_{p}\right)
$$

where $\mathbf{G}_{\chi}(\tilde{\chi})$ is the FE solution of problem (13) for a particular value of the dropper lengths and $G_{l}\left(l_{p}\right)$ is a constant function with unitary value in all $\Omega_{l}$. Considering $\mathbf{R}_{1}(\tilde{\chi})=$ $\mathbf{G}_{\chi}(\tilde{\chi})$ and $L_{1}\left(l_{p}\right)=G_{l}\left(l_{p}\right)$, the remaining functions $\mathbf{R}_{i}(\tilde{\chi})$ and $L_{i}\left(l_{p}\right)$, for $i>1$, are calculated with homogeneous Dirichlet boundary conditions. The interested reader is referred to $[25,26]$ for a detailed explanation of the PGD construction algorithm for problems with homogeneous Dirichlet boundary conditions.

The solution enrichment process starts from the assumed computed $\mathbf{r}^{n}, n \geq 1$, and then the next term of the separated solution is sought

$$
\mathbf{r}^{n+1}=\sum_{i=1}^{n} \mathbf{R}_{i}(\tilde{\chi}) L_{i}\left(l_{p}\right)+\mathbf{R}_{n+1}(\tilde{\chi}) L_{n+1}\left(l_{p}\right)
$$

with an admissible variation

$$
\delta \mathbf{r}=\delta \mathbf{R}_{n+1} L_{n+1}+\mathbf{R}_{n+1} \delta L_{n+1}
$$


Introducing (20) and (21) in (17) leads to a nonlinear expression in which $\mathbf{R}_{n+1}$ and $L_{n+1}$ are the unknown functions. A widely used procedure to find these functions is based on an alternating fixed point strategy [26, 25, 27]. In each iteration of the proposed algorithm, two problems have to be solved in order to obtain both the $\mathbf{R}_{n+1}(\tilde{\chi})$ and $L_{n+1}\left(l_{p}\right)$ functions. These problems are detailed in Appendix A and briefly described below:

- Calculation of $\mathbf{R}_{n+1}(\tilde{\chi})$. In this problem, $\mathbf{r}^{n}$ is known and $L_{n+1}\left(l_{p}\right)$ comes from either the previous iteration of the alternating strategy or it is randomly chosen in the case of the first iteration. Introducing Eq. (20) into (17) and integrating in $\Omega_{l}$, $\mathbf{R}_{n+1}(\tilde{\chi})$ is the only unknown of the problem. Consider that

$$
\delta \mathbf{r}=\delta \mathbf{R}_{n+1} L_{n+1}
$$

is the test function. The element contribution to the static equilibrium is

$$
\begin{aligned}
& \int_{0}^{1}\left[E I \delta \mathbf{R}^{\prime \prime} \cdot\left(\omega_{i} \mathbf{R}_{i}^{\prime \prime}+\pi \mathbf{R}_{n+1}^{\prime \prime}\right)+\frac{E A}{2} \delta \mathbf{R}^{\prime} \cdot\left[\alpha_{i j k} \mathbf{R}_{i}^{\prime}\left(\mathbf{R}_{j}^{\prime} \cdot \mathbf{R}_{k}^{\prime}\right)+\right.\right. \\
& \beta_{i j}\left(2 \mathbf{R}_{i}^{\prime}\left(\mathbf{R}_{j}^{\prime} \cdot \mathbf{R}_{n+1}^{\prime}\right)+\mathbf{R}_{n+1}^{\prime}\left(\mathbf{R}_{i}^{\prime} \cdot \mathbf{R}_{j}^{\prime}\right)\right)+ \\
& \gamma_{i}\left(2 \mathbf{R}_{n+1}^{\prime}\left(\mathbf{R}_{i}^{\prime} \cdot \mathbf{R}_{n+1}^{\prime}\right)+\mathbf{R}_{i}^{\prime}\left(\mathbf{R}_{n+1}^{\prime} \cdot \mathbf{R}_{n+1}^{\prime}\right)\right)+ \\
& \left.\left.\theta \mathbf{R}_{n+1}^{\prime}\left(\mathbf{R}_{n+1}^{\prime} \cdot \mathbf{R}_{n+1}^{\prime}\right)-\omega_{i} \mathbf{R}_{i}^{\prime}-\eta \mathbf{R}_{n+1}^{\prime}\right]-\tau \rho A \delta \mathbf{R} \cdot \mathbf{g}\right] d \tilde{\chi}
\end{aligned}
$$

where $\mathbf{R}^{\prime}$ and $\mathbf{R}^{\prime \prime}$ are the first and second derivatives of $\mathbf{R}$ respect to the variable $\tilde{\chi}$, and the repeated subscripts $i, j, k$ represent summations from 1 to $\mathrm{n}$, following the Einstein notation. The coefficients $\alpha_{i j k}, \beta_{i j}, \gamma_{i}, \theta, \omega_{i}, \eta$ and $\tau$ are the integrals in $\Omega_{l}$ described in Appendix A. Finally, this second order nonlinear boundary value problem is solved for $\mathbf{R}_{n+1}(\tilde{\chi})$ using the FEM discretization described in Section 3.

- Calculation of $L_{n+1}\left(l_{p}\right)$. Now in this problem, $\mathbf{r}^{n}$ is again known and $\mathbf{R}_{n+1}(\tilde{\chi})$ is the just-computed function. Introducing Eq. (20) into (17) and integrating in $\tilde{\chi}$, $L_{n+1}\left(l_{p}\right)$ is the only unknown of the problem. In this case:

$$
\delta \mathbf{r}=\mathbf{R}_{n+1} \delta L_{n+1}
$$


is the test function and leads to the element contribution:

$$
\begin{aligned}
& \int_{\Omega_{l}}\left[\frac { \delta L } { l _ { p } } \left[I_{2 i} L_{i}+I_{1} L_{n+1}+\frac{1}{l_{p}^{2}}\left(\alpha_{i j k} L_{i} L_{j} L_{k}+\left(\beta_{1 i j}+\beta_{2 i j}\right) L_{n+1} L_{i} L_{j}+\right.\right.\right. \\
& \left.\left.\left.\left(\gamma_{1 i}+\gamma_{2 i}\right) L_{n+1}^{2} L_{i}+\theta L_{n+1}^{3}\right)-\omega_{i} L_{i}-\eta L_{n+1}\right]-\delta L \tau l_{p}\right] d l_{p}
\end{aligned}
$$

where the coefficients $I_{1}, I_{2 i}, \alpha_{i j k}, \beta_{1 i j}, \beta_{2 i j}, \gamma_{1 i}, \gamma_{2 i}, \theta, \omega_{i}, \eta$ and $\tau$ are the integrals in the variable $\tilde{\chi}$ listed in Appendix A. Finally, a nonlinear algebraic problem is obtained, which in this paper is solved for $L_{n+1}\left(l_{p}\right)$ using an appropriate discretization technique.

Both functions obtained at fixed point iteration $p$ are compared with the same functions at the previous step $p-1$. The iterative process proceeds until its relative difference becomes smaller than a certain prefixed value $\varepsilon$, i.e.

$$
\frac{\left\|\mathbf{R}_{n+1}^{p} L_{n+1}^{p}-\mathbf{R}_{n+1}^{p-1} L_{n+1}^{p-1}\right\|}{\left\|\mathbf{R}_{n+1}^{p-1} L_{n+1}^{p-1}\right\|}<\varepsilon
$$

The solution enrichment process ends when the error $\Sigma(n)$ is small enough, $\Sigma(n)<\tilde{\varepsilon}$. Among the existing stopping criteria, for its simplicity and short calculation time, we chose

$$
\Sigma(n)=\frac{\left\|\mathbf{R}_{n} L_{n}\right\|}{\left\|\mathbf{R}_{k} L_{k}\right\|}
$$

where $k$ is 2 if the first mode is employed to enforce the non-homogeneous Dirichlet boundary conditions, or 1 otherwise.

When the just described separated representation constructor is used in nonsymmetric problems, the obtained solution contains more modes than those provided by the Singular Value Decomposition (SVD) (or its multidimensional counterpart, the High Order Singular Value Decomposition (HOSVD)) applied to the problem solution computed by using standard discretization techniques. Thus, the decomposition provided by the PGD constructor is not optimal [28]. This reveals that some modes do not make an important contribution to the solution reconstruction and are not necessary in the reduced basis.

Therefore, a post-compression should be envisaged in order to express the solution in a more compact form (see [29] and [26]). If $\mathbf{r}^{\hat{n}}$ is the PGD solution of the original problem 
with $\hat{n}$ modes, the post-compression is carried out by solving the following problem:

$$
\int_{\Omega_{l}} \int_{\Omega} \delta \mathbf{r}_{c}\left[\mathbf{r}_{c}\left(\tilde{\chi}, l_{p}\right)-\mathbf{r}^{\hat{n}}\left(\tilde{\chi}, l_{p}\right)\right] d \tilde{\chi} d l_{p}=0
$$

where $\mathbf{r}_{c}\left(\tilde{\chi}, l_{p 1}\right)$ is the unknown field. The PGD is here applied only for approximation purposes. With this technique the number of modes of the approximated solution $\mathbf{r}_{c}^{n}$ are usually fewer than those making up the original solution, i.e. $n \leq \hat{n}$. Therefore, post-processing the PGD solution requires a lighter computational effort.

\subsection{Generalization to $\mathscr{N}$ extra-coordinates}

It is straightforward to extrapolate the previous method to a more general case in which there are $\mathscr{N}$ different undeformed dropper lengths extra-coordinates. In this case, the domain $\Omega_{l}=\Omega_{l_{1}} \times \Omega_{l_{2}} \times \ldots \times \Omega_{l_{N}}$ where $\Omega_{l_{i}}=\left[l_{p i}^{-}, l_{p i}^{+}\right], i=1, \ldots, \mathscr{N}$. The separated representation of the absolute position field is:

$$
\mathbf{r}\left(\tilde{\chi}, l_{p 1}, \ldots, l_{p \mathscr{N}}\right) \approx \mathbf{r}^{n}=\sum_{i=1}^{n} \mathbf{R}_{i}(\tilde{\chi}) \prod_{j=1}^{\mathscr{N}} L_{i j}\left(l_{p j}\right)
$$

Assuming $\mathbf{r}^{n}\left(\tilde{\chi}, l_{p 1}, \ldots, l_{p \mathscr{N}}\right)$ is known, the next term of the separated representation is:

$$
\mathbf{r}^{n+1}=\mathbf{r}^{n}+\mathbf{R}_{n+1}(\tilde{\chi}) \prod_{j=1}^{\mathscr{N}} L_{n+1, j}\left(l_{p j}\right)
$$

and the virtual displacements field can be chosen as:

$$
\delta \mathbf{r}=\delta \mathbf{R}_{n+1} \prod_{j=1}^{\mathscr{N}} L_{i j}\left(l_{p j}\right)+\mathbf{R}_{n+1} \sum_{i=1}^{\mathscr{N}} \delta L_{n+1, i} \prod_{\substack{j=1 \\ j \neq i}}^{\mathscr{N}} L_{n+1, j}
$$

Replacing Eqs. (30) and (31) in (17) and applying the fixed point iterative strategy, the resolution of $\mathscr{N}+1$ one dimensional problems at each fixed point iteration must be performed in order to calculate the functions $\mathbf{R}_{n+1}(\tilde{\chi}), L_{n+1,1}, \ldots, L_{n+1, \mathcal{N}}$ respectively. 


\subsection{PGD formulation: Dropper slackening}

In the formulation presented in the previous section the droppers are allowed to be in compression due to the forces applied by the messenger and contact wires. However, this is not strictly true, since the droppers can slacken when the pantograph pushes them upwards. In the case of the static configuration problem, they may have some combinations of undeformed dropper lengths that result in the slackening of some droppers in the static equilibrium position. Although this is not desirable in a railway catenary, if the model could take it into account it would be possible to simulate mistakes in the assembly process or failures in the design.

In a classic FEM approach it is easy to account for this effect by neglecting the axial strain term in Eq. (13) for the droppers whose deformed length is shorter than undeformed length. When the problem is solved by the PGD approach the solution to this issue is not straightforward. Here, we propose the following procedure to capture dropper slackening in the separate solution:

1. Solve the PGD problem described in Section 5.1, in which droppers can be compressed.

2. For each dropper $p$, identify the combinations of extra-coordinates in which the dropper is compressed. For this pupose, the function $h^{p} \in \Omega_{l}$ is defined, which takes the value of 1 when the dropper is stretched and is equal to 0 otherwise.

3. If there is more than one extra-coordinate, separate each $h^{p}$ function in a summation of the product of one dimensional functions:

$$
h^{p}=\sum_{i=1}^{N_{p}} H_{i 1}\left(l_{p 1}\right) H_{i 2}\left(l_{p 2}\right) \ldots H_{i \mathscr{N}}\left(l_{p \mathscr{N}}\right)
$$

4. Solve a PGD static problem in which, for dropper $p$, the axial strain term of the element contribution to the static equilibrium (17) is multiplied by its separate $h^{p}$ function.

In practice, in a real catenary with a realistic range of variation of dropper lengths, if we focus on a single dropper, its slackening can be assumed to depend only on its initial length 
and the undeformed length of its two neighbors. With this assumption, each $h^{p}$ function depends, at most, on three extra-coordinates. Otherwise, in problems with several extracoordinates, it would be challenging to explore all $\Omega_{l}$ to identify the dropper slackening due to computational memory limitations.

Let us assume that there are $P$ droppers in the spatial domain $\Omega$, and for simplicity in the notation, the undeformed length of the first two droppers are considered as extracoordinates, namely $l_{p 1}$ and $l_{p 2}$. By solving the problem posted in Section 5.1 but now with these two extra-coordinates, the separated absolute position field is obtained:

$$
\mathbf{r}_{P G D}\left(\tilde{\chi}, l_{p 1}, l_{p 2}\right)=\sum_{i=1}^{N} \mathbf{R}_{i}(\tilde{\chi}) L_{i 1}\left(l_{p 1}\right) L_{i 2}\left(l_{p 2}\right)
$$

Next, for each dropper $p$ the domain $\Omega_{l}=\Omega_{l_{1}} \times \Omega_{l_{2}}$ is explored and the $h^{p}$ function is built. Specifically, for dropper 2:

$$
h^{2}\left(l_{p 1}, l_{p 2}, l_{03}\right)=\left\{\begin{array}{lll}
1 & \text { if } & \frac{l_{\text {def }}}{l_{l_{2}}} \geq 1 \\
0 & \text { if } & \frac{l_{d e f}}{l_{p 2}}<1
\end{array}\right.
$$

where $l_{\text {def }}$ is the deformed length of dropper 2 and $l_{03}$ denotes the undeformed length of dropper 3, which is assumed to be constant in this case.

Following the procedure described in Appendix B, all of these $h^{p}$ functions are exactly represented with $N_{p}$ modes in a separate form:

$$
h^{p}\left(l_{p 1}, l_{p 2}\right)=\sum_{i=1}^{N_{p}} H_{i 1}\left(l_{p 1}\right) H_{i 2}\left(l_{p 2}\right)
$$

The last step of the proposed strategy consists of solving a PGD static equilibrium problem with the use of the $h^{p}$ functions, in order to cancel the axial force of the droppers for all the combinations of $l_{p 1}$ and $l_{p 2}$ that make them slacken. The procedure for obtaining the solution to this problem is exactly the same as described in the previous Section 5.1. However, in this case, for a dropper $p$ with variable initial length $l_{p 1}$, its contribution to the static equilibrium (Eq. (17)) becomes:

$$
\int_{\Omega_{l}} \int_{0}^{1}\left[H_{m 1}\left(l_{p 1}\right) H_{m 2}\left(l_{p 2}\right) \frac{E A}{2 l_{p 1}^{2}} \frac{d \delta \mathbf{r}}{d \tilde{\chi}} \cdot \frac{d \mathbf{r}}{d \tilde{\chi}}\left(\frac{1}{l_{p 1}^{2}} \frac{d \mathbf{r}}{d \tilde{\chi}} \cdot \frac{d \mathbf{r}}{d \tilde{\chi}}-1\right)-\delta \mathbf{r} \cdot \mathbf{g}\right] l_{p 1} d \tilde{\chi} d l_{p 1} d l_{p 2}
$$


where the subscript $m$ denotes a summation. From this expression, we proceed just as in Section 5.1 and compute the separated representation of the absolute position field.

This method leads to accurate results in spite of having some drawbacks. For example, when the high nonlinearity of the dropper slackening is introduced into the problem, the fixed-point alternating strategy needs more iterations to converge, with a consequently higher computational cost. Moreover, looking at expression (36), when the separated field (20) is introduced there is a summation whose number of terms depends cubically on the previous computed modes, and linearly on the number of terms in the separated $h^{p}$ function.

It is true that these calculations are performed offline and only once, however they can require excessive computational time. For this reason, we propose the linearization of the problem (13) with respect to one static equilibrium position computed from a determined dropper lengths. The linear solution is close to the nonlinear solution, as will be seen in Section 6, and the time required to obtain this solution is orders of magnitude lower.

\subsection{PGD formulation: Linearized static problem}

In a classic FEM approach, in order to linearize the static equilibrium problem, the first step is to calculate the static equilibrium configuration for which the problem is linearized. This involves solving the problem stated in (13) for a certain values of undeformed dropper lengths $\mathbf{l}_{r e f}$ defining the reference catenary spatial domain $\Omega_{r e f}$. This provides $\mathbf{r}_{r e f}(\chi)$, the reference equilibrium position field. For a different set of undeformed dropper lengths $\mathbf{l}_{0}$ defining the spatial domain $\Omega$, the new equilibrium position can be expressed as $\mathbf{r}=$ $\mathbf{r}_{r e f}+\mathbf{u}$, where $\mathbf{u}(\chi)$ is the displacement field with respect to the reference solution $\mathbf{r}_{r e f}(\chi)$. Therefore, the linearized static equilibrium problem consists of solving for all admissible $\delta \mathbf{u}$ :

$$
\begin{aligned}
\sum_{e=1}^{N_{e}} \int_{\Omega^{e}} & {\left[E I \frac{d^{2} \delta \mathbf{u}}{d \chi^{2}} \cdot \frac{d^{2} \mathbf{u}}{d \chi^{2}}+\frac{E A}{2} \frac{d \delta \mathbf{u}}{d \chi} \cdot\left[2 \frac{d \mathbf{r}_{r e f}}{d \chi}\left(\frac{d \mathbf{u}}{d \chi} \cdot \frac{d \mathbf{r}_{r e f}}{d \chi}\right)+\frac{d \mathbf{u}}{d \chi}\left(\frac{d \mathbf{r}_{r e f}}{d \chi} \cdot \frac{d \mathbf{r}_{r e f}}{d \chi}\right)+\right.\right.} \\
+ & \left.\left.\frac{d \mathbf{r}_{r e f}}{d \chi}\left(\frac{d \mathbf{r}_{r e f}}{d \chi} \cdot \frac{d \mathbf{r}_{r e f}}{d \chi}\right)-\frac{d \mathbf{u}}{d \chi}-\frac{d \mathbf{r}_{r e f}}{d \chi}\right]-\rho A \delta \mathbf{u} \cdot \mathbf{g}\right] d \chi=0, \quad \forall \delta \mathbf{u}
\end{aligned}
$$


In order to apply the PGD to such a variational problem, the reference solution $\mathbf{r}_{r e f}(\chi)$, which only depends on the spatial coordinate, is obtained again solving Eq. (13), in which $\mathbf{l}_{r e f}$ are now defined as the intermediate values of the undeformed dropper lengths in $\Omega_{l}$. From now on, the same steps as detailed in Section 5.1 must be followed. The displacements field also depends on all the extra-coordinates, so the change of variable defined in (16) is introduced. Taking only one extra-coordinate for simplicity in the notation, the element contribution to the linearized static equilibrium problem reads:

$$
\begin{aligned}
& \int_{\Omega_{l}} \int_{0}^{1} {\left[\frac{E I}{l_{p}^{3}} \frac{d^{2} \delta \mathbf{u}}{d \tilde{\chi}^{2}} \cdot \frac{d^{2} \mathbf{u}}{d \tilde{\chi}^{2}}+\frac{E A}{2} \frac{d \delta \mathbf{u}}{d \tilde{\chi}} \cdot\left[\frac{2}{l_{p}^{3}} \frac{d \mathbf{r}_{r e f}}{d \tilde{\chi}}\left(\frac{d \mathbf{u}}{d \tilde{\chi}} \cdot \frac{d \mathbf{r}_{r e f}}{d \tilde{\chi}}\right)+\frac{1}{l_{p}^{3}} \frac{d \mathbf{u}}{d \tilde{\chi}}\left(\frac{d \mathbf{r}_{r e f}}{d \tilde{\chi}} \cdot \frac{d \mathbf{r}_{r e f}}{d \tilde{\chi}}\right)+\right.\right.} \\
&\left.\left.+\frac{1}{l_{p}^{3}} \frac{d \mathbf{r}_{r e f}}{d \tilde{\chi}}\left(\frac{d \mathbf{r}_{r e f}}{d \tilde{\chi}} \cdot \frac{d \mathbf{r}_{r e f}}{d \tilde{\chi}}\right)-\frac{1}{l_{p}} \frac{d \mathbf{u}}{d \tilde{\chi}}-\frac{1}{l_{p}} \frac{d \mathbf{r}_{r e f}}{d \tilde{\chi}}\right]-\rho A l_{p} \delta \mathbf{u} \cdot \mathbf{g}\right] d \tilde{\chi} d l_{p}
\end{aligned}
$$

The separated form of the unknown field is:

$$
\mathbf{u}\left(\tilde{\chi}, l_{p}\right) \approx \mathbf{u}^{n}=\sum_{i=1}^{N} \mathbf{U}_{i}(\tilde{\chi}) L_{i}\left(l_{p}\right)
$$

where $\mathbf{U}(\tilde{\chi})$ is a function that only depends on the normalized spatial coordinate $\tilde{\chi}$, and $L\left(l_{p}\right)$ is a function which depends on the extra-coordinate $l_{p}$. As the Dirichlet boundary conditions are fulfilled by the reference static solution $\mathbf{r}_{r e f}$, the linearized problem is solved with homogeneous Dirichlet boundary conditions.

In order to obtain the next mode $\mathbf{u}^{n+1}$, the procedure used is again the same as described in Section 5.1. In order to account for the dropper slackening, the procedure exlained in Section 5.3 is applied combined with the linearized problem defined above, considering now that Eq.(34) becomes:

$$
h^{p}\left(l_{p 1}, l_{p 2}\right)=\left\{\begin{array}{lll}
1 & \text { if } \quad f_{\text {int }}^{p} \geq 0 \\
0 & \text { if } \quad f_{\text {int }}^{p}<0
\end{array}\right.
$$

where $f_{i n t}^{p}$ is the projected internal force of the element $p$ in its direction.

By using the linearized formulation, when solving the problem defined in $\Omega \times \Omega_{l}$ with the fixed-point algorithm, the number of terms involved in the internal forces increases linearly with the number of already evaluated modes. On the other hand, if the nonlinear 
formulation is used, the number of terms increases cubically with the evaluated modes. This means there is a considerable reduction in computational cost when the linearized formulation is employed.

\section{Numerical examples}

In this section, the proposed method is checked through some numerical examples. The first one is quite simple, allowing all the FEM static solutions to be obtained and thus making it possible to compare the whole nonlinear PGD solution with the solutions obtained from the FE analysis. The second one includes a more realistic catenary, with whose geometry a realistic application of the algorithm is shown. Finally, the third example reveals that when the nonlinear problem formulation includes dropper slackening, the solution process is very expensive in terms of computational cost, so that the linearized approach is used and validated.

\subsection{Example 1: Academic example}

The proposed method is validated with the first numerical example. The spatial domain $\Omega$ of this $2 \mathrm{D}$ academic example is shown in Fig.4. It simulates a single span with two droppers, in which the contact and messenger wires are discretized into 30 elements each. The material and geometrical properties of these elements are listed in Table 1. Regarding the Dirichlet boundary conditions, the vertical and horizontal displacements of the nodes located at the ends of both cables are constrained. Altogether, there are 240 spatial degrees of freedom. The two droppers are considered with variable undeformed length. The domains of the extra-coordinates $l_{p 1}$ and $l_{p 2}$ are $\Omega_{l_{1}}=\Omega_{l_{2}}=[1.15,1.25] \mathrm{m}$, which ensure dropper slackening in certain regions of $\Omega_{l}$. The domains of each extra-coordinate are discretized into 20 elements of the same length.

The first PGD solution is obtained allowing the dropper elements to work in compression. The iterative process converges with 12 modes. Fast convergence of fixed-point alternating strategy was observed (around 8 iterations each mode) and short computational time was required. 


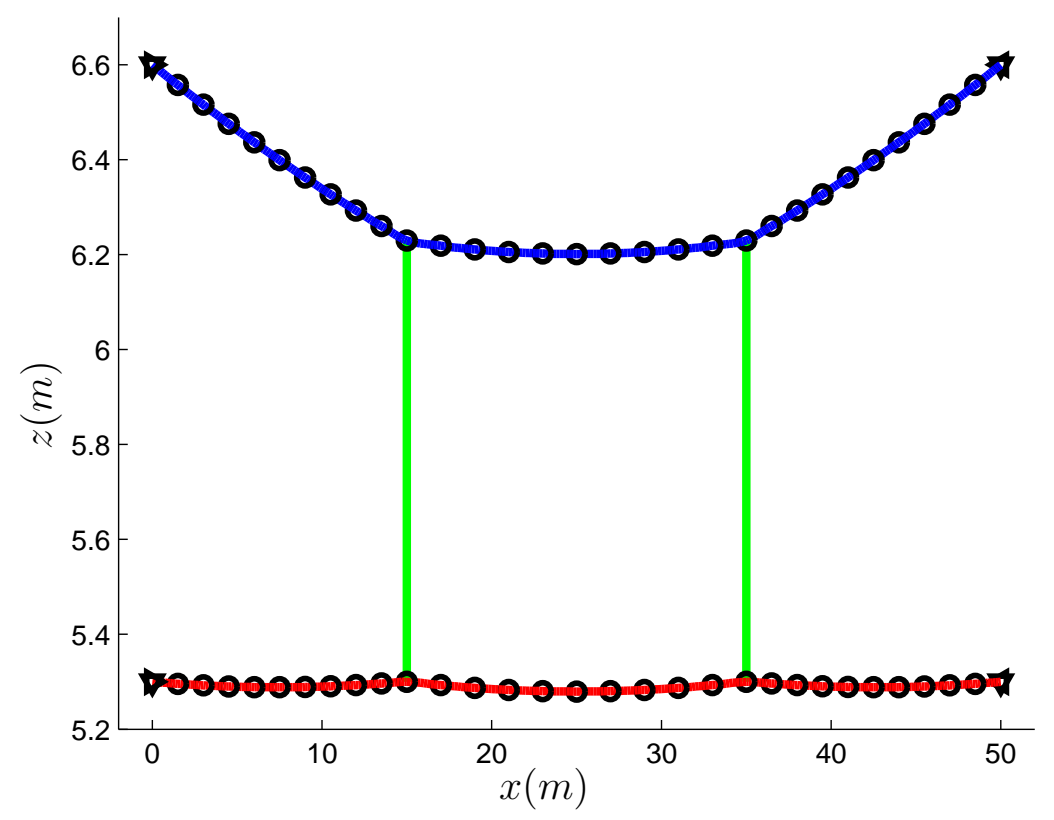

Figure 4: Academic example spatial mesh.

\begin{tabular}{|c|c|c|c|c|}
\hline Element & $\mathbf{E}(\mathrm{Pa})$ & $\boldsymbol{\rho}\left(\mathrm{kg} / \mathrm{m}^{3}\right)$ & $\mathbf{A}\left(\mathrm{mm}^{2}\right)$ & $\mathbf{I}\left(\mathrm{mm}^{4}\right)$ \\
\hline \hline Messenger wire & 9114 & $1.1 \cdot 10^{11}$ & 94.8 & 1237.2 \\
\hline Contact wire & 9160 & $1.1 \cdot 10^{11}$ & 150 & 2170 \\
\hline Droppers & 9114 & $1.1 \cdot 10^{11}$ & 10 & 0 \\
\hline
\end{tabular}

Table 1: Material and geometrical properties of the elements.

From this solution, the $h^{1}$ and $h^{2}$ functions are calculated. It should be remembered that the $h^{p}$ function controls whether or not the dropper $p$ is under compression $(h=0)$ or $(h=1)$ for any combination of variable undeformed dropper lengths. These functions are plotted in Fig. 5. The dark grey area is the region of the domain $\Omega_{l}$, in which the respective dropper is stretched, while the light grey area shows the values of the extracoordinates at which the dropper is compressed. Focusing on $h^{1}$, it can be seen that when dropper 1 becomes longer it tends to slacken, while on the other hand, dropper 1 is more likely to slacken when dropper 2 becomes shorter. The same conclusions can be drawn for dropper 2, looking at $h^{2}$.

The separation of $h^{1}$ results in a summation of 9 terms and $h^{2}$ is separated properly with 10 modes. The next step of the calculation is to compute the PDG solution, taking dropper slackening into account. The strong nonlinearity introduced by the $h^{p}$ functions slows 

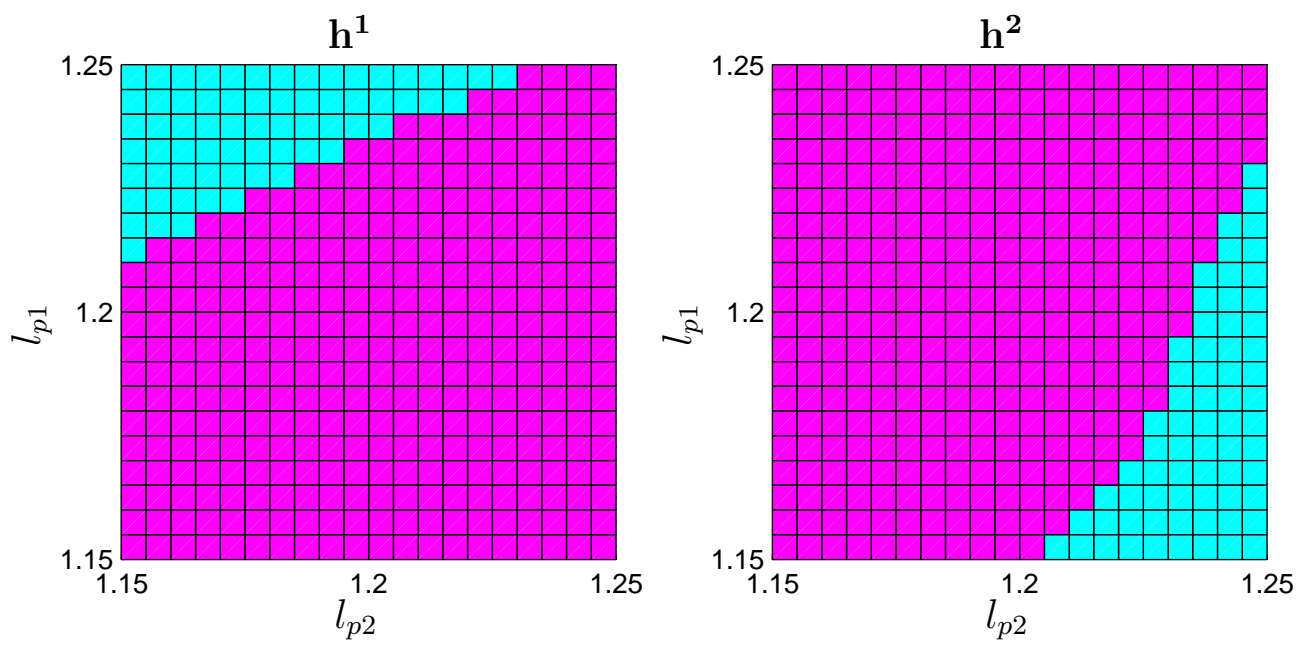

Figure 5: $h$ function for droppers 1 and 2. (Light grey) Blue $=0$, (Dark grey) Magenta $=1$. down the speed of convergence of the fixed-point strategy. Furthermore, the number of modes necessary to obtain a similar error to the case of droppers without slacking increases hugely, as can be seen in Fig. 6. For these reasons, the computational cost required to solve this problem is much higher than that required to solve the PGD problem without dropper slackening.

Let us define the local error of the separated solution with $N$ modes and for specific values of the extra-coordinates, as:

$$
\begin{aligned}
E_{l o c}^{N}\left(l_{p 1}, l_{p 2}, \ldots, l_{p \mathscr{N}}\right) & =\frac{\left\|\mathbf{r}_{E X}\left(l_{p 1}, l_{p 2}, \ldots, l_{p \mathscr{N}}\right)-\mathbf{r}_{P G D}^{N}\left(l_{p 1}, l_{p 2}, \ldots, l_{p \mathscr{N}}\right)\right\|}{\left\|\mathbf{r}_{E X}\left(l_{p 1}, l_{p 2}, \ldots, l_{p \mathscr{N}}\right)\right\|} \\
& =\frac{\sqrt{\sum_{i=1}^{N_{e}} \int_{0}^{l_{0}}\left|\mathbf{r}_{E X}^{\mathbf{i}}\left(l_{p 1}, l_{p 2}, \ldots, l_{p \mathscr{N}}\right)-\mathbf{r}_{P G D}^{\mathbf{i}^{N}}\left(l_{p 1}, l_{p 2}, \ldots, l_{p \mathscr{N}}\right)\right|^{2} d \chi}}{\sqrt{\sum_{i=1}^{N_{e}} \int_{0}^{l_{0}}\left|\mathbf{r}_{E X}^{\mathbf{i}}\left(l_{p 1}, l_{p 2}, \ldots, l_{p \mathscr{N}}\right)\right|^{2} d \chi}}
\end{aligned}
$$

where $\mathbf{r}_{E X}$ and $\mathbf{r}_{P G D}^{N}$ are the absolute position field from the FEM solution and the PGD solution, respectively. To account for all $\Omega_{l}$, a global error is defined as the integral of the local error in this domain:

$$
E_{g l o b}^{N}=\int_{\Omega_{l}} E_{l o c}^{N}\left(l_{p 1}, l_{p 2}, \ldots, l_{p \mathscr{N}}\right) d l_{p 1} d l_{p 2} \ldots d l_{p \mathscr{N}}
$$


Fig. 6 shows the global error versus the number of PGD modes. The graph on the left shows the case with dropper slackening and the one on the right gives the error for the problem without dropper slackening. As mentioned above, the speed of convergence to the reference FEM solution is noticeably slower than the case without dropper slackening.

The 27 computed modes of the separated solution can be compressed into a few modes using (28) with almost the same accuracy, as shown in Fig. 7.
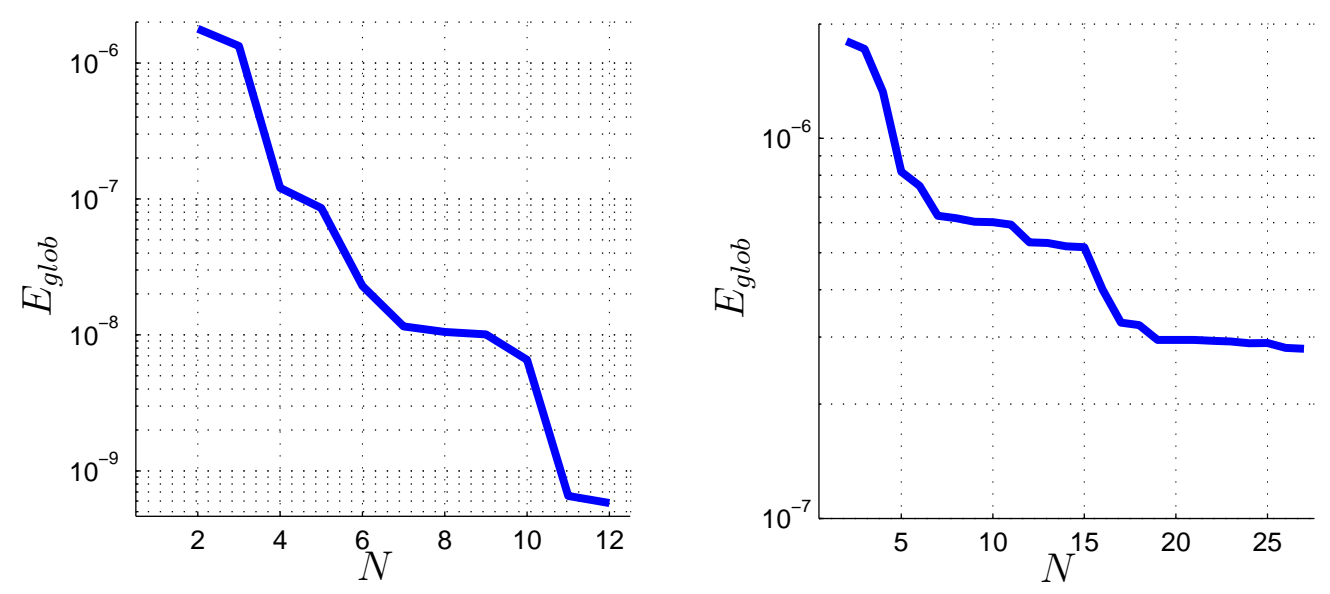

Figure 6: $E_{\text {glob }}$ of PGD solution with dropper slackening (left) and without dropper slackening (right).

Fig. 8 shows a comparison between the solutions considering (dark grey line) and obviating (light grey line) the slackening of dropper 1 . In both cases, the values of the undeformed lengths are $l_{p 1}=1.25 \mathrm{~m}$ and $l_{p 2}=1.15 \mathrm{~m}$. The difference in the static equilibrium position is quite clear, hence the necessity of including dropper slackening into the PGD approach to obtain realistic solutions.

\subsection{Example 2: Variable presag}

In this case, a real span of an overhead line set-up is studied. The span consists of 9 droppers, the messenger wire and the contact wire discretized into 100 elements each. As 


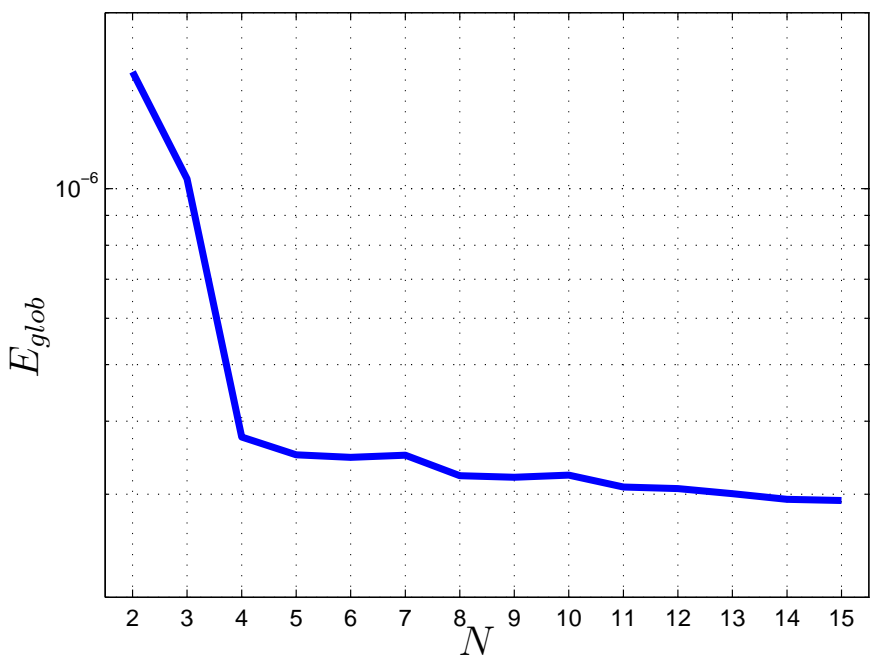

Figure 7: $E_{\text {glob }}$ of PGD post-compressed solution.

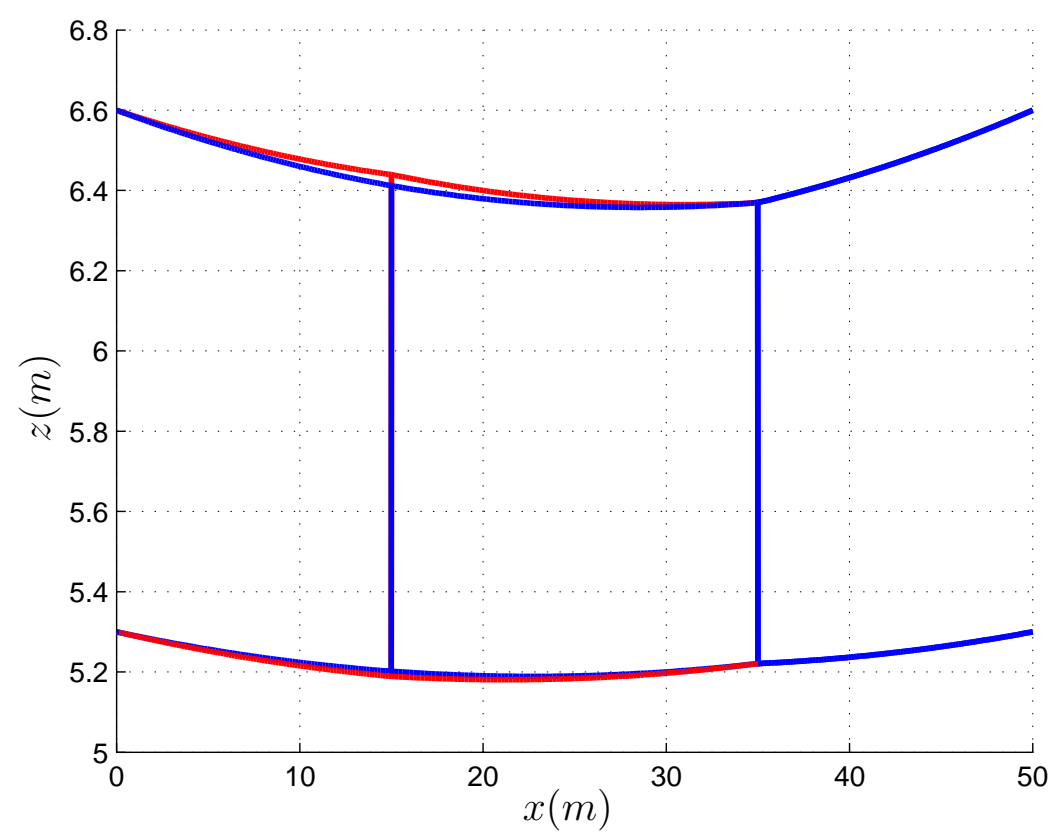

Figure 8: Static position for $l_{p 1}=1.25 \mathrm{~m}$ and $l_{p 2}=1.15 \mathrm{~m}$. With dropper slackening (blue/dark grey), without dropper slackening (red/light grey).

in the previous example, the vertical and horizontal displacements of the nodes at the ends of both cables are constrained.

In a real railway catenary the presence of a certain amount of presag is fairly common. 
Presag is defined as the vertical distance at the central point of the span, in the static equilibrium configuration, from the contact wire to the horizontal, as shown in Fig. 9.

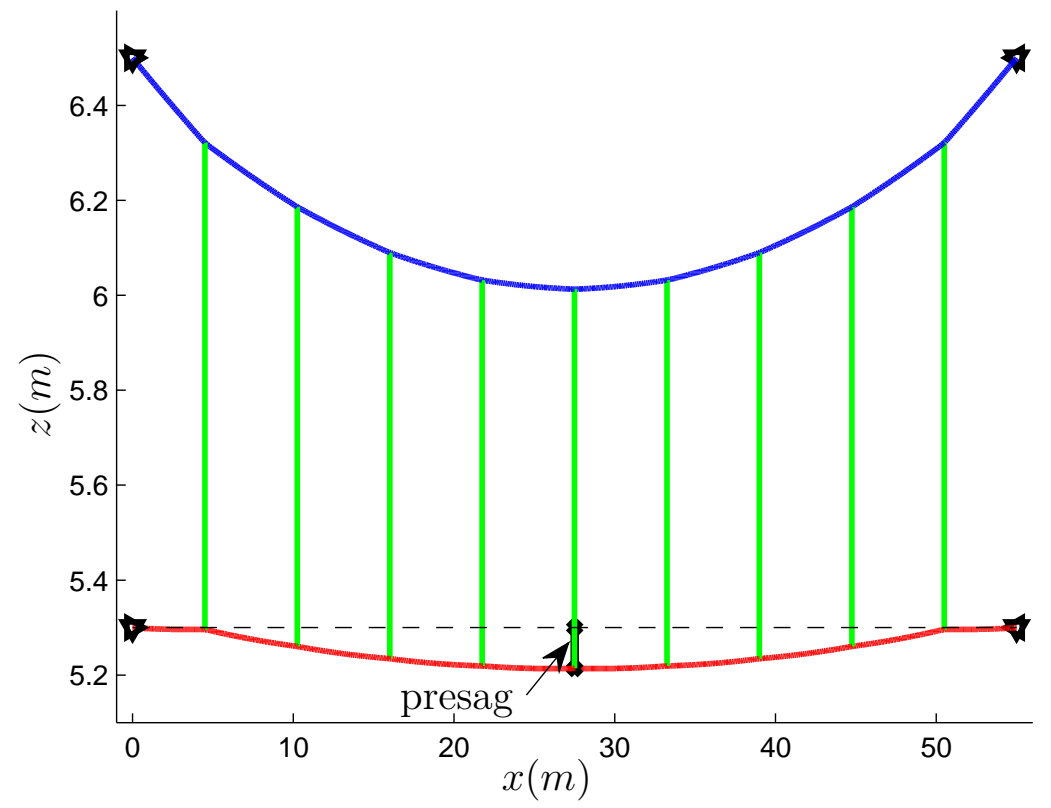

Figure 9: Geometric model of a real span, with defined presag.

The method described in this paper not only allows the undeformed dropper lengths to be included as extra-coordinates, but also allows the extra-coordinate to be a parameter on which the undeformed dropper lengths depend, i.e. for dropper $i, l_{p i}=f_{i}$ (parameter). Since the amount of presag is defined with the initial dropper lengths, it is possible to include an extra-coordinate of the problem that controls the presag. Each undeformed length of droppers 2 to 8 is variable and depends on the $x$ coordinate of the dropper, on the undeformed dropper length that leads to a static position in which the presag is null, and finally on the new variable $p s g \in \Omega_{p s g}=[0,0.18] \mathrm{m}$. This range of values of the extra-coordinate $p s g$ is equivalent to a presag of $[0,85] \mathrm{mm}$ measured in the equilibrium position. The domain of the extra-coordinate is discretized into 20 uniform distributed elements.

The PGD solution to this problem, in which there is no dropper slackening, is accurate enough with 5 modes, as showed in Fig. 10.

In Fig. 11 the $L_{i}(p s g), i>1$ normalized functions are plotted. It is notorious that 


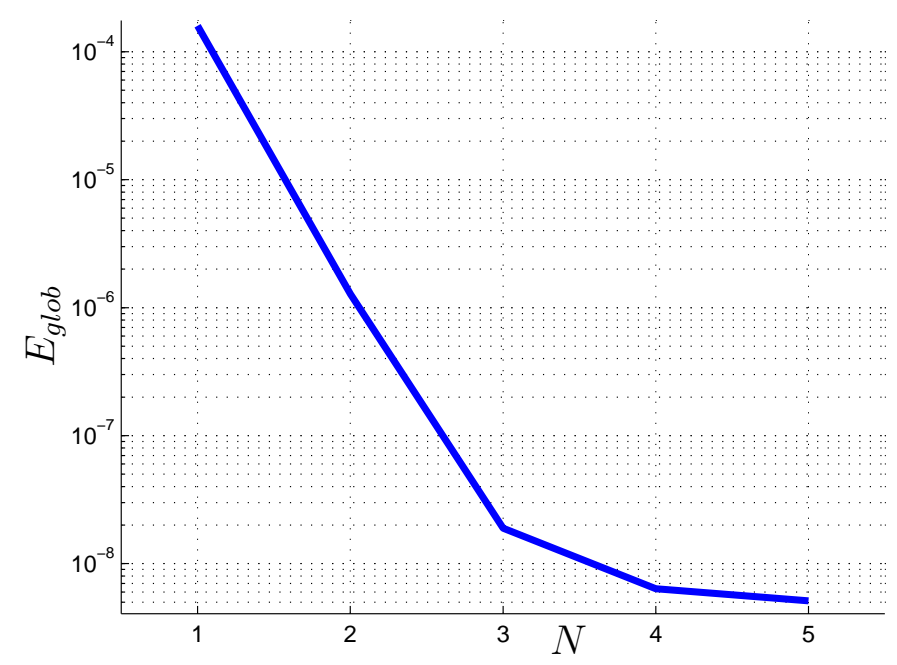

Figure 10: Global error for PGD solution with variable presag.

$L_{i}(0)=0, i=2, \ldots, 5$. This is because the first mode $R_{1}(\tilde{\chi}) L_{1}(p s g)$ introduced into the solution to fulfill the Dirichlet boundary conditions is the FEM solution of the static equilibrium problem for zero presag. Therefore, as expected, the following PGD modes do not change the solution for $p s g=0$.

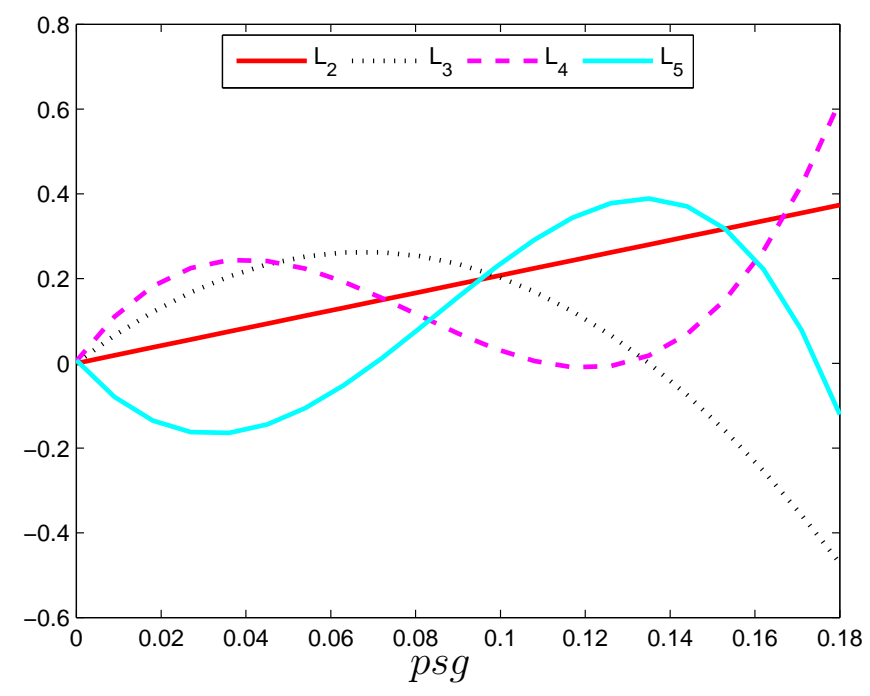

Figure 11: $L_{i}(p s g), i>1$ normalized functions.

Once the solution is obtained, we have to check if there are any compressed droppers. In the case in hand, all the $h^{p}$ functions are null, thus all droppers are under tension in all 


\begin{tabular}{|c|c|c|}
\hline Dropper & Extra-coordinate & Range $(\mathbf{m})$ \\
\hline \hline $1-9$ & $l_{p 1}$ & $1.00-1.05$ \\
\hline $2-8$ & $l_{p 2}$ & $0.82-0.87$ \\
\hline $3-7$ & $l_{p 3}$ & $0.70-0.75$ \\
\hline $4-6$ & $l_{p 4}$ & $0.62-0.68$ \\
\hline 5 & $l_{p 5}$ & $0.59-0.64$ \\
\hline
\end{tabular}

Table 2: Definition of the domains for each extra-coordinate.

$\Omega_{l}$. This example shows what is expected to take place in a real railway catenary static configuration. The values of the initial dropper lengths are designed so that in the static equilibrium configuration all the droppers work under tension. This PGD solution could therefore be used as the initial modes of the solution of a dynamic simulation problem in the PGD approach.

\subsection{Example 3: Multidimensional catenary span}

The same geometrical model of the catenary span was used in this case as in the variable presag example. The nine droppers in the span are considered as elements with variable initial length. In particular, as regards the span symmetry, the symmetric droppers are related to the same extra-coordinate, and so five extra-coordinates are introduced. The range of variation of each variable and the droppers related to them are listed in Table 2 . The $5 \mathrm{D}$ domain $\Omega_{l}$ is composed of the product of the $1 \mathrm{D}$ domains $\Omega_{l i}$, which are discretized into 20 uniform elements each.

The first step deals with the PGD problem without dropper slackening, using the nonlinear formulation of the static equation. In the present case, due to its high dimensionality, it is not possible to generate a reference solution with classical methods, so that the local and global error cannot be evaluated. As all the $L_{i j}(i=1, \ldots, N ; j=1, \ldots, 5)$ functions are normalized, the weight of the mode $n$ is defined as $w_{n}=\left\|\mathbf{R}_{n}\right\|$. Fig. 12 represents the weight of the first 30 modes, where its decreasing tendency can be seen.

When building the $h^{p}$ functions the nine droppers are compressed for certain $\Omega_{l}$ regions. Therefore, the next step is to solve the static equilibrium problem, including dropper slackening. However, even if this is performed offline, using the nonlinear formulation takes an excessive time. Consequently, when there are several extra-coordinates, the 


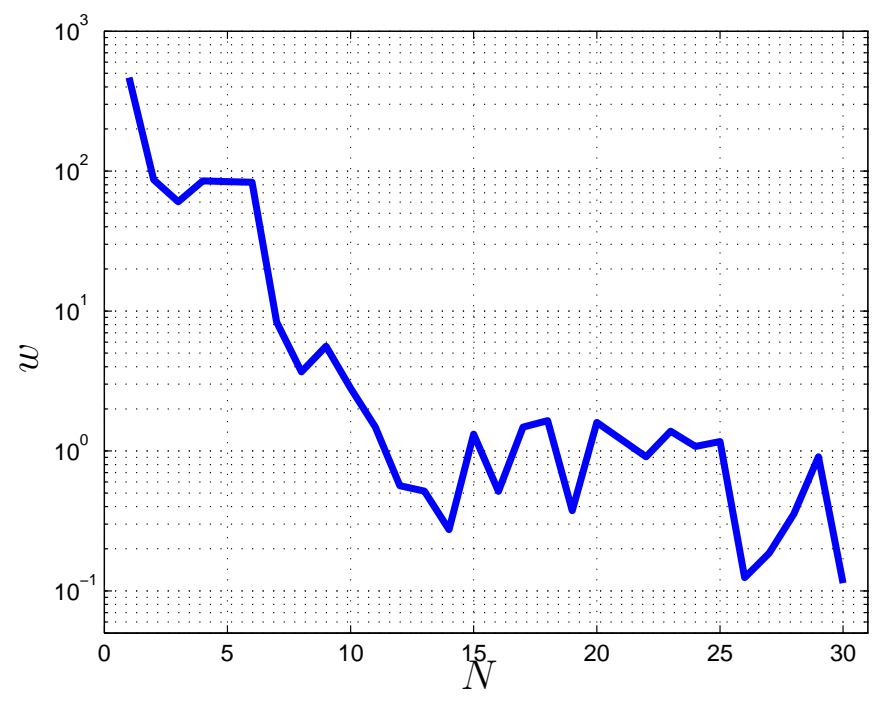

Figure 12: Weight of the PGD modes of the nonlinear problem without dropper slackening.

linearized formulation is highly advantageous in terms of computational cost without affecting the required accuracy.

Fig. 13 shows a comparison of the static equilibrium position solving the nonlinear and the linearized problem. The maximum values are used for the $l_{p}$ coordinates, so that this case is the furthest case from the reference solution, i.e. the distance between $\Omega_{r e f}$ and $\Omega$ is maximum for the current $\Omega_{l}$. Even so, the largest differences observed in the height of the contact wire are around $1 \mathrm{~mm}$. These discrepancies are perfectly assumable and confirm the validity of the linearized static formulation for this $\Omega_{l}$.

When computing the PGD solution with dropper slackening, using the linearized formulation of the problem, 225 modes are computed to achieve good accuracy in the separate solution. This number of modes would be challenging to obtain with the nonlinear formulation, whilst it requires little computational cost with the linearized problem.

Fig. 14 shows the weights of the computed modes for the original solution (left) and the post-compressed solution (right). Although there are some peaks, the general trend is to reduce the weight of the modes while the PGD solution tends to the exact solution of the problem. This tendency is clearly improved when the post-compressor problem (28) is solved. However, for the established accuracy, the number of modes required is not reduced when the solution is compressed, which means that every mode plays an important 


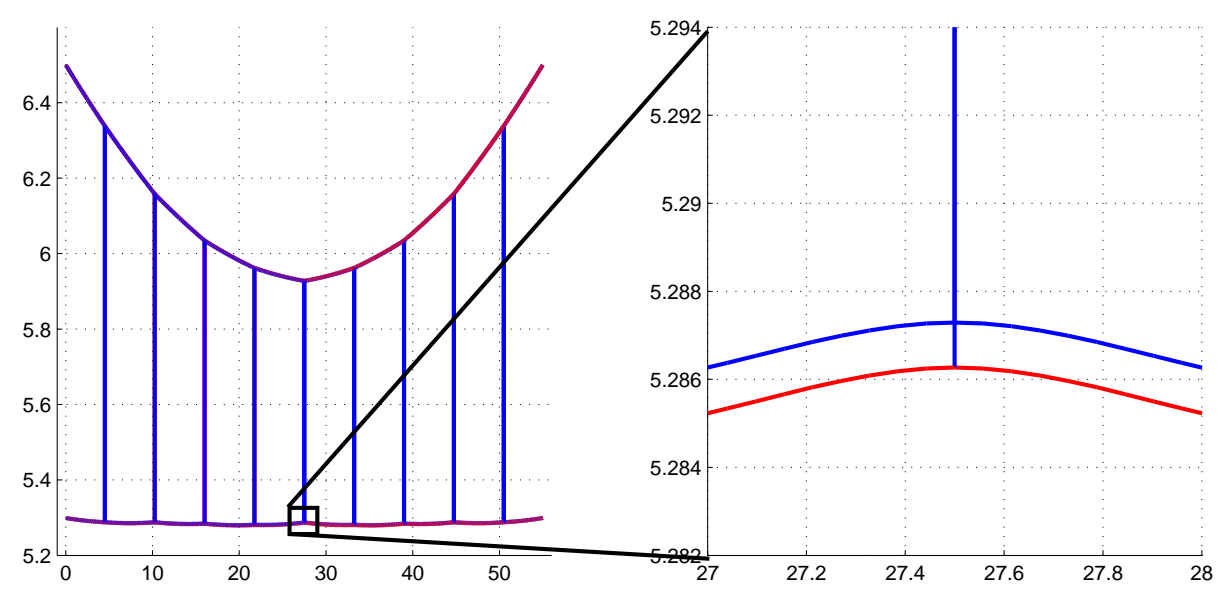

Figure 13: Comparison of linear and nonlinear static equilibrium positions.

role in the reconstruction of the solution.
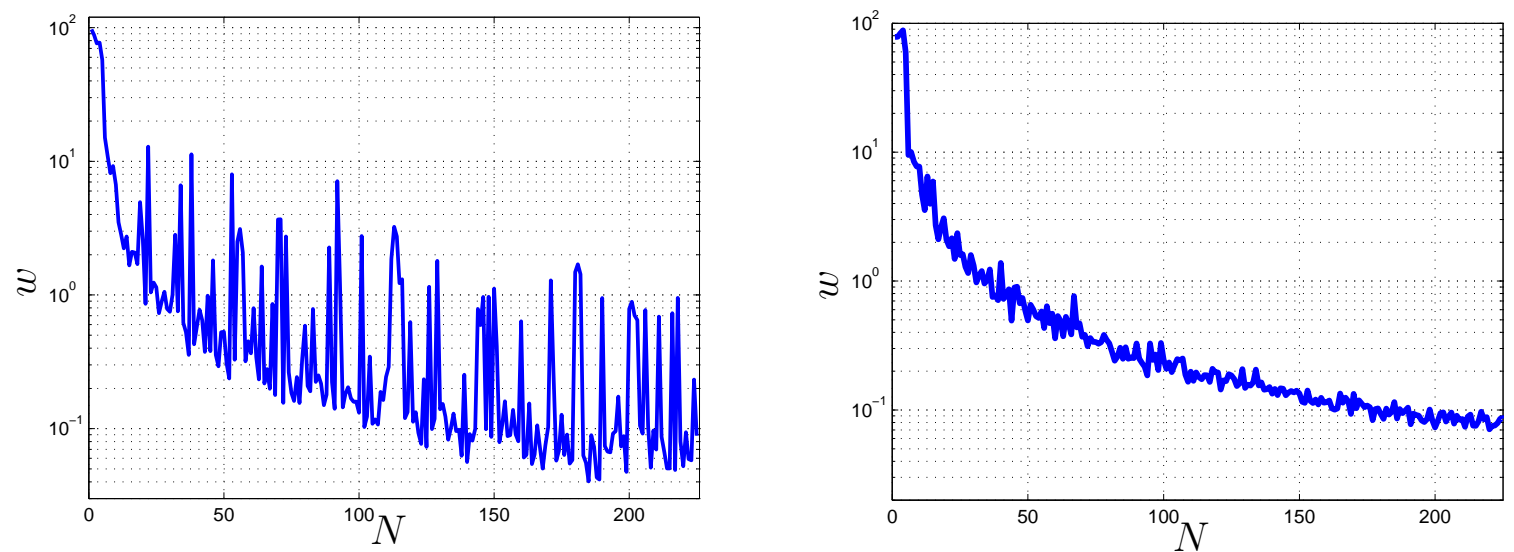

Figure 14: Weight of the PGD modes of the linear problem solution, with slackening. Original solution (left), post-compressed solution (right).

Finally, in order to emphasize the effect of considering dropper slackening, Fig. 15 shows the static equilibrium configuration for $l_{p 1}=1 \mathrm{~m}, l_{p 2}=0.82 \mathrm{~m}, l_{p 3}=0.75 \mathrm{~m}, l_{p 4}=0.62 \mathrm{~m}$ and $l_{p 5}=0.64 \mathrm{~m}$. On the dark grey plot, droppers $2,4,6$ and 8 are compressed, while on the light grey plot they are slackened. 


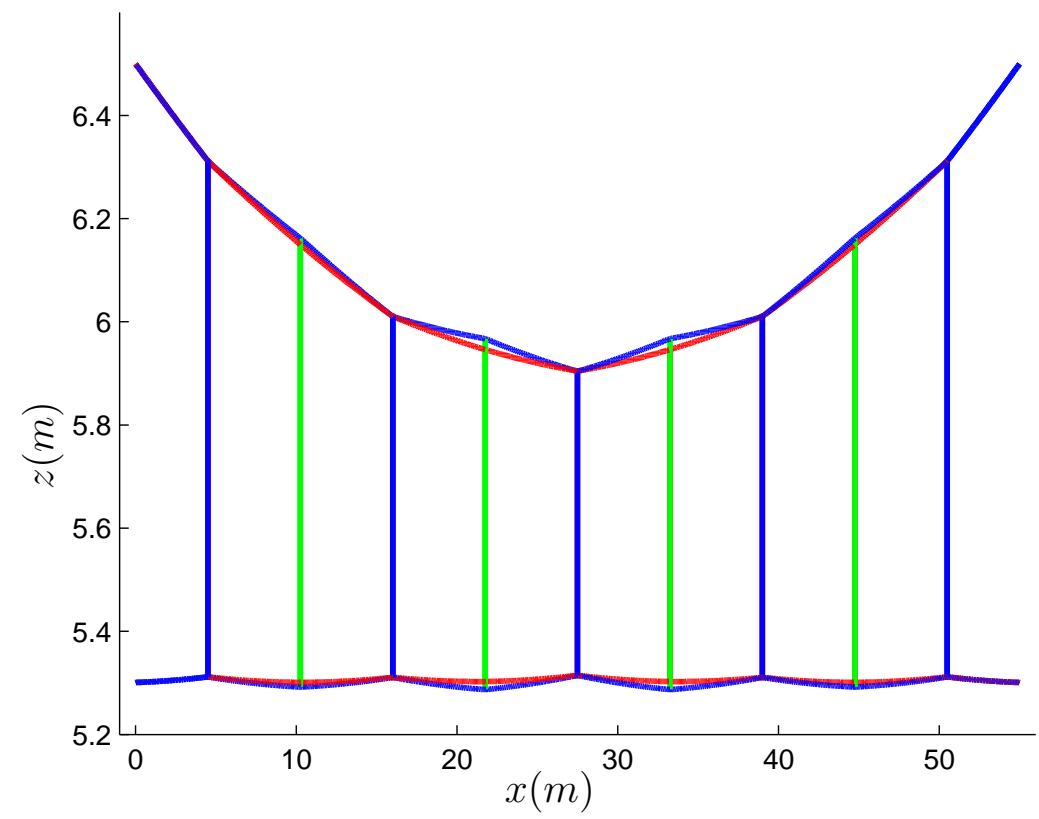

Figure 15: Static equilibrium with compressed droppers and slackened droppers.

\section{Conclusions}

This paper addresses the static equilibrium problem of a railway catenary. The catenary system was modeled by FE according to the absolute nodal coordinate formulation. The undeformed dropper lengths were introduced into the nonlinear formulation as extracoordinates in order to obtain a general solution valid for any value of undeformed dropper length, using PGD. The proposed strategy was applied to an academic example and showed good convergence and accuracy. Not only can undeformed dropper lengths be added as extra-coordinates, but also any parameter on which these lengths depend. In order to demonstrate the method's capabilities, presag was included as an extra-coordinate in the model. The proposed method also allows for the strong nonlinearity of dropper slackening. This nonlinear effect has a remarkable influence in the number of modes needed for the solution and thus increases the computational time. In order to make the offline part of the process more efficient, a linearized formulation of the problem was therefore proposed and provided a similar level of accuracy in the results. The method was also applied to a realistic catenary model. 
This work constitutes the first step towards the major objective of geometric catenary optimization, which normally requires a large number of dynamic catenary simulations for different values of the parameters to be optimized, e.g. undeformed dropper lengths. With the parametric solution provided by the PGD approach, i.e. the dynamic response of the system for any combination of undeformed dropper lengths, addressing the optimization problem would be faster because one dynamic simulation would be substituted by a particularization of the parametric solution. However, in order to obtain the parametric dynamic solution, it is necessary to have at hand a parametric solution of the static configuration problem presented in this paper.

\section{Appendix A. The PGD constructor}

The aim of this section is to explain in depth the formulation of the two problems dealt with in Section 5.1, in which the functions $\mathbf{R}_{n+1}(\tilde{\chi})$ and $L_{n+1}\left(l_{p}\right)$ are sought.

\section{A.1. Computing $\mathbf{R}_{n+1}(\tilde{\chi})$ from $L_{n+1}\left(l_{p}\right)$}

For this problem, the test function is:

$$
\delta \mathbf{r}=\delta \mathbf{R}(\tilde{\chi}) L_{n+1}\left(l_{p}\right)
$$

where $L_{n+1}\left(l_{p}\right)$ in known. Introducing equations (20) and (43) into (17) the following expression is obtained:

$$
\begin{aligned}
& \int_{[0,1] \times \Omega_{l}}\left[\frac{E I}{l_{p}^{2}} L_{n+1} \delta \mathbf{R}^{\prime \prime} \cdot\left(\mathbf{R}_{i}^{\prime \prime} L_{i}+\mathbf{R}_{n+1}^{\prime \prime} L_{n+1}\right)+\right. \\
& \frac{E A}{2 l_{p}^{2}} L_{n+1} \delta \mathbf{R}^{\prime} \cdot\left(\mathbf{R}_{i}^{\prime} L_{i}+\mathbf{R}_{n+1}^{\prime} L_{n+1}\right)\left[\frac{1}{l_{p}^{2}}\left(\mathbf{R}_{j}^{\prime} L_{j}+\mathbf{R}_{n+1}^{\prime} L_{n+1}\right) \cdot\left(\mathbf{R}_{k}^{\prime} L_{k}+\mathbf{R}_{n+1}^{\prime} L_{n+1}\right)-1\right]- \\
& \left.L_{n+1} \delta \mathbf{R} \rho \mathbf{g} A\right] l_{p} d \tilde{\chi} d l_{p}
\end{aligned}
$$


Integrating in $\Omega_{l}$, the coefficients:

$$
\begin{array}{lll}
\alpha_{i j k}=\int_{\Omega_{l}} \frac{1}{l_{p}^{3}} L_{n+1} L_{i} L_{j} L_{k} d l_{p} & \beta_{i j}=\int_{\Omega_{l}} \frac{1}{l_{p}^{3}} L_{n+1}^{2} L_{i} L_{j} d l_{p} & \gamma_{i}=\int_{\Omega_{l}} \frac{1}{l_{p}^{3}} L_{n+1}^{3} L_{i} d l_{p} \\
\theta=\int_{\Omega_{l}} \frac{1}{l_{p}^{3}} L_{n+1}^{4} d l_{p} & \omega_{i}=\int_{\Omega_{l}} \frac{1}{l_{p}} L_{n+1} L_{i} d l_{p} & \eta=\int_{\Omega_{l}} \frac{1}{l_{p}} L_{n+1}^{2} d l_{p} \\
\tau=\int_{\Omega_{l}} L_{n+1} l_{p} d l_{p} & &
\end{array}
$$

can be introduced into Eq.(44) and results in:

$$
\begin{aligned}
& \int_{0}^{1}\left[E I \delta \mathbf{R}^{\prime \prime} \cdot\left(\omega_{i} \mathbf{R}_{i}^{\prime \prime}+\pi \mathbf{R}_{n+1}^{\prime \prime}\right)+\frac{E A}{2} \delta \mathbf{R}^{\prime} \cdot\left[\alpha_{i j k} \mathbf{R}_{i}^{\prime}\left(\mathbf{R}_{j}^{\prime} \cdot \mathbf{R}_{k}^{\prime}\right)+\right.\right. \\
& \beta_{i j}\left(2 \mathbf{R}_{i}^{\prime}\left(\mathbf{R}_{j}^{\prime} \cdot \mathbf{R}_{n+1}^{\prime}\right)+\mathbf{R}_{n+1}^{\prime}\left(\mathbf{R}_{i}^{\prime} \cdot \mathbf{R}_{j}^{\prime}\right)\right)+\gamma_{i}\left(2 \mathbf{R}_{n+1}^{\prime}\left(\mathbf{R}_{i}^{\prime} \cdot \mathbf{R}_{n+1}^{\prime}\right)+\mathbf{R}_{i}^{\prime}\left(\mathbf{R}_{n+1}^{\prime} \cdot \mathbf{R}_{n+1}^{\prime}\right)\right)+ \\
& \left.\left.\theta \mathbf{R}_{n+1}^{\prime}\left(\mathbf{R}_{n+1}^{\prime} \cdot \mathbf{R}_{n+1}^{\prime}\right)-\omega_{i} \mathbf{R}_{i}^{\prime}-\eta \mathbf{R}_{n+1}^{\prime}\right]-\tau \rho A \delta \mathbf{R} \cdot \mathbf{g}\right] d \tilde{\chi}
\end{aligned}
$$

Discretizing the function $\mathbf{R}$ and applying the Galerkin FEM, a nonlinear system of algebraic equations is obtained which can be solved with the Newton-Raphson method.

\section{A.2. Computing $L_{n+1}\left(l_{p}\right)$ from $\mathbf{R}_{n+1}(\tilde{\chi})$}

For the next step of the fixed-point iterative process, the test function is chosen as

$$
\delta \mathbf{r}=\mathbf{R}_{n+1}(\tilde{\chi}) \delta L\left(l_{p}\right)
$$

where $\mathbf{R}_{n+1}(\tilde{\chi})$ is the solution of the problem solved in the previous step. In this case, the contribution of an element to the problem at hand is:

$$
\begin{aligned}
& \int_{[0,1] \times \Omega_{l}}\left[\frac{E I}{l_{p}^{2}} \delta L \mathbf{R}_{n+1}^{\prime \prime} \cdot\left(\mathbf{R}_{i}^{\prime \prime} L_{i}+\mathbf{R}_{n+1}^{\prime \prime} L_{n+1}\right)+\right. \\
& \frac{E A}{2 l_{p}^{2}} \delta L \mathbf{R}_{n+1}^{\prime} \cdot\left(\mathbf{R}_{i}^{\prime} L_{i}+\mathbf{R}_{n+1}^{\prime} L_{n+1}\right) \cdot\left[\frac{1}{l_{p}^{2}}\left(\mathbf{R}_{j}^{\prime} L_{j}+\mathbf{R}_{n+1}^{\prime} L_{n+1}\right) \cdot\left(\mathbf{R}_{k}^{\prime} L_{k}+\mathbf{R}_{n+1}^{\prime} L_{n+1}\right)-1\right]- \\
& \left.\rho A \delta L \mathbf{R}_{n+1} \cdot \mathbf{g}\right] l_{p} d \tilde{\chi} d l_{p}
\end{aligned}
$$


Integrating the known functions in the corresponding domain and defining the following coefficients:

$$
\begin{array}{ll}
I_{1}=E I \int_{0}^{1} \mathbf{R}_{n+1}^{\prime \prime} \cdot \mathbf{R}_{n+1}^{\prime \prime} d \tilde{\chi} & I_{2 i}=E I \int_{0}^{1} \mathbf{R}_{n+1}^{\prime \prime} \cdot \mathbf{R}_{i}^{\prime \prime} d \tilde{\chi} \\
\alpha_{i j k}=\frac{E A}{2} \int_{0}^{1}\left(\mathbf{R}_{n+1}^{\prime} \cdot \mathbf{R}_{i}^{\prime}\right)\left(\mathbf{R}_{j}^{\prime} \cdot \mathbf{R}_{k}^{\prime}\right) d \tilde{\chi} & \beta_{1 i j}=\frac{E A}{2} \int_{0}^{1}\left(\mathbf{R}_{n+1}^{\prime} \cdot \mathbf{R}_{n+1}^{\prime}\right)\left(\mathbf{R}_{i}^{\prime} \cdot \mathbf{R}_{j}^{\prime}\right) d \tilde{\chi} \\
\beta_{2 i j}=\frac{E A}{2} \int_{0}^{1}\left(\mathbf{R}_{n+1}^{\prime} \cdot \mathbf{R}_{i}^{\prime}\right)\left(\mathbf{R}_{n+1}^{\prime} \cdot \mathbf{R}_{j}^{\prime}\right) d \tilde{\chi} & \gamma_{1 i}=\frac{E A}{2} \int_{0}^{1}\left(\mathbf{R}_{n+1}^{\prime} \cdot \mathbf{R}_{i}^{\prime}\right)\left(\mathbf{R}_{n+1}^{\prime} \cdot \mathbf{R}_{n+1}^{\prime}\right) d \tilde{\chi} \\
\gamma_{2 i}=\frac{E A}{2} \int_{0}^{1}\left(\mathbf{R}_{n+1}^{\prime} \cdot \mathbf{R}_{n+1}^{\prime}\right)\left(\mathbf{R}_{i}^{\prime} \cdot \mathbf{R}_{n+1}^{\prime}\right) d \tilde{\chi} & \theta=\frac{E A}{2} \int_{0}^{1}\left(\mathbf{R}_{n+1}^{\prime} \cdot \mathbf{R}_{n+1}^{\prime}\right)^{2} d \tilde{\chi} \\
\omega_{i}=\frac{E A}{2} \int_{0}^{1} \mathbf{R}_{n+1}^{\prime} \cdot \mathbf{R}_{i}^{\prime} d \tilde{\chi} & \eta=\frac{E A}{2} \int_{0}^{1} \mathbf{R}_{n+1}^{\prime} \cdot \mathbf{R}_{n+1}^{\prime} d \tilde{\chi} \\
\tau=\rho A \int_{0}^{1} \mathbf{R}_{n+1} \cdot \mathbf{g} d \tilde{\chi} &
\end{array}
$$

Eq. (48) is rewritten in the following fashion:

$$
\begin{aligned}
& \int_{\Omega_{l}}\left[\frac { \delta L } { l _ { p } } \left[I_{2 i} L_{i}+I_{1} L_{n+1}+\frac{1}{l_{p}^{2}}\left(\alpha_{i j k} L_{i} L_{j} L_{k}+\left(\beta_{1 i j}+\beta_{2 i j}\right) L_{n+1} L_{i} L_{j}+\right.\right.\right. \\
& \left.\left.\left.\left(\gamma_{1 i}+\gamma_{2 i}\right) L_{n+1}^{2} L_{i}+\theta L_{n+1}^{3}\right)-\omega_{i} L_{i}-\eta L_{i}\right]-\delta L \tau l_{p}\right] d l_{p}
\end{aligned}
$$

which can be solved using an appropriate discretization technique. However, this expression can be turned into its associated nonlinear strong form:

$$
\begin{aligned}
& \frac{1}{l_{p}}\left[I_{2 i} L_{i}+I_{1} L_{n+1}+\frac{1}{l_{p}^{2}}\left(\alpha_{i j k} L_{i} L_{j} L_{k}+\left(\beta_{1 i j}+\beta_{2 i j}\right) L_{n+1} L_{i} L_{j}+\right.\right. \\
& \left.\left.\left(\gamma_{1 i}+\gamma_{2 i}\right) L_{n+1}^{2} L_{i}+\theta L_{n+1}^{3}\right)-\omega_{i} L_{i}-\eta L_{n+1}\right]-\tau l_{p}
\end{aligned}
$$

The nonlinear system equation resulting from the result of both strategies can be solved again using the Newton-Raphson method.

\section{Appendix B. Separation of the $h$ function}

Let $h^{p}$ be a function dependent on three variables, $h^{p}\left(l_{p 1}, l_{p 2}, l_{p 3}\right)$. An example of such a function is depicted in Fig. 16. The domain $\Omega_{l}$ is discretized into $N_{1} \times N_{2} \times N_{3}$ points 
in which $h^{p}$ takes the value of one (shadowed square vertices) or zero (other points). The goal of the separation process is to obtain the functions $H_{1}\left(l_{p 1}\right), H_{2}\left(l_{p 2}\right)$ and $H_{3}\left(l_{p 3}\right)$ such that:

$$
h^{p}\left(l_{p 1}, l_{p 2}, l_{p 3}\right)=\sum_{i=1}^{N_{p}} H_{1 i}\left(l_{p 1}\right) H_{2 i}\left(l_{p 2}\right) H_{3 i}\left(l_{p 3}\right)
$$

All these functions are step unitary functions. Defining the residual $R_{e} s_{i}$ as the difference of $h^{p}$ and the right hand side of Eq. (52) for a certain value of $i$, the proposed algorithm reads:

1. Initialize $i=1, l_{p 2}=l_{p 2}^{-}$and $l_{p 3}=l_{p 3}^{-}$.

2. Evaluate Res $_{i}$. Three cases are distinguished:

(a) If all values are 0 , there is not a new term. Go to Step 3.

(b) If all values are 1 , the new term is composed of $H_{1 i}\left(l_{p 1}\right)=1, H_{2 i}\left(l_{p 2}^{-}\right)=1$ and $H_{3 i}\left(l_{p 3}^{-}\right)=1$. Update $i=i+1$, and go to Step 3 .

(c) If some values of $\operatorname{Res}_{i}$ are 1 and others are $0, H_{1 i}\left(l_{p 1}\right)=1$. For the other two directions $R_{e} s_{i}$ is evaluated starting from the first nonzero value of the function $H_{1 i}$ (black point). Each function of the new term corresponds to the evaluation of $R_{e} s_{i}$ in its direction. Update $i=i+1$, and go to Step 3.

3. Move to the next value of $l_{p 2}$ and/or $l_{p 3}$ and repeat Step 1 until all the domain is explored. In this case, move to Step 4.

4. All the modes where $H_{1 i}\left(l_{p 1}\right)=1$ are compressed in an appropriate way in order to minimize the number of terms in (52).

\section{Acknowledgments}

The authors would like to acknowledge the financial support of the FPU program offered by the Ministerio de Educación, Cultura y Deporte under grant number (FPU13/04191). The funding from Universitat Politècnica de València and Generalitat Valenciana (PROMETEO/2012/023) are also acknowledged. 


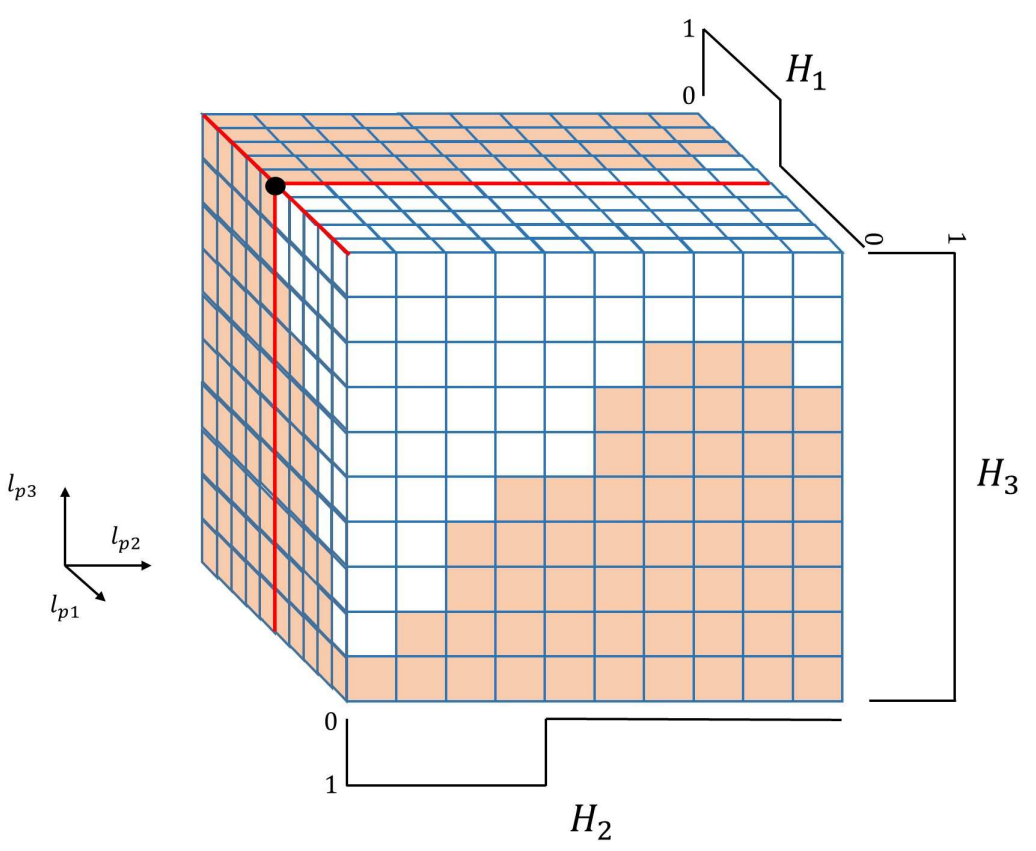

Figure 16: Example of $h$ function and separation method. The value of $h$ in the corners of the shadowed cubes are 1 and in the rest is 0 .

\section{References}

[1] S. Bruni, J. Ambrosio, A. Carnicero, Y. H. Cho, L. Finner, M. Ikeda, S. Y. Kwon, J.-P. Massat, S. Stichel, M. Tur, et al., "The results of the pantograph-catenary interaction benchmark," Vehicle System Dynamics, vol. 53, no. 3, pp. 412-435, 2015.

[2] M. Tur, L. Baeza, F. Fuenmayor, and E. García, "PACDIN statement of methods," Vehicle System Dynamics, vol. 53, no. 3, pp. 402-411, 2015.

[3] A. Facchinetti and S. Bruni, "Special issue on the pantograph-catenary interaction benchmark," Vehicle System Dynamics, vol. 53, no. 3, pp. 303-304, 2015.

[4] W. Zhang, G. MET, and J. Zeng, "A study of pantograph/catenary system dynamics with influence of presag and irregularity of contact wire," Vehicle system dynamics, vol. 37, pp. 593-604, 2002. 
[5] Y. H. Cho, K. Lee, Y. Park, B. Kang, and K.-n. Kim, "Influence of contact wire pre-sag on the dynamics of pantograph-railway catenary," International journal of mechanical sciences, vol. 52, no. 11, pp. 1471-1490, 2010.

[6] A. Ammar, B. Mokdad, F. Chinesta, and R. Keunings, "A new family of solvers for some classes of multidimensional partial differential equations encountered in kinetic theory modeling of complex fluids," Journal of Non-Newtonian Fluid Mechanics, vol. 139 , pp. 153-176, dec 2006.

[7] B. Bognet, A. Leygue, and F. Chinesta, "On the fully 3D simulations of thermoelastic models defined in plate and shell geometries," European Journal of Computational Mechanics/Revue Européenne de Mécanique Numérique, vol. 21, no. 1-2, pp. 40-51, 2012.

[8] B. Bognet, A. Leygue, and F. Chinesta, "Separated representations of 3D elastic solutions in shell geometries," Advanced Modeling and Simulation in Engineering Sciences, vol. 1, no. 1, pp. 1-4, 2014.

[9] A. Ammar, A. Huerta, F. Chinesta, E. Cueto, and A. Leygue, "Parametric solutions involving geometry: A step towards efficient shape optimization," Computer Methods in Applied Mechanics and Engineering, vol. 268, pp. 178-193, jan 2014.

[10] F. Chinesta, A. Ammar, A. Leygue, and R. Keunings, "An overview of the proper generalized decomposition with applications in computational rheology," Journal of Non-Newtonian Fluid Mechanics, vol. 166, no. 11, pp. 578-592, 2011.

[11] E. Giner, B. Bognet, J. J. Ródenas, A. Leygue, F. J. Fuenmayor, and F. Chinesta, "The Proper Generalized Decomposition (PGD) as a numerical procedure to solve 3D cracked plates in linear elastic fracture mechanics," International Journal of Solids and Structures, vol. 50, pp. 1710-1720, may 2013.

[12] S. Niroomandi, D. González, I. Alfaro, F. Bordeu, A. Leygue, E. Cueto, and F. Chinesta, "Real-time simulation of biological soft tissues: a PGD approach.," International journal for numerical methods in biomedical engineering, vol. 29, pp. 586600, may 2013. 
[13] S. Niroomandi, I. Alfaro, D. González, E. Cueto, and F. Chinesta, "Model order reduction in hyperelasticity: a proper generalized decomposition approach," International Journal for Numerical Methods in Engineering, pp. n/a-n/a, jul 2013.

[14] L. Boucinha, A. Gravouil, and A. Ammar, "Space-time proper generalized decompositions for the resolution of transient elastodynamic models," Computer Methods in Applied Mechanics and Engineering, vol. 255, pp. 67-88, 2013.

[15] J. Almeida, "A basis for bounding the errors of proper generalised decomposition solutions in solid mechanics," International Journal for Numerical Methods in Engineering, vol. 94, no. 10, pp. 961-984, 2013.

[16] F. Chinesta, R. Keunings, and A. Leygue, The Proper Generalized Decomposition for Advanced Numerical Simulations: A Primer. Springer Publishing Company, Incorporated, oct 2013.

[17] M. Tur, E. García, L. Baeza, and F. Fuenmayor, "A 3D absolute nodal coordinate finite element model to compute the initial configuration of a railway catenary," Engineering Structures, vol. 71, pp. 234-243, 2014.

[18] A. Shabana, "Computer implementation of the absolute nodal coordinate formulation for flexible multibody dynamics," Nonlinear Dynamics, vol. 16, pp. 293-306, 1998.

[19] M. Berzeri and A. Shabana, "Development of simple models for the elastic forces in the absolute nodal coordinate formulation," Journal of Sound and Vibration, vol. 235, no. 4, pp. 539-565, 2000.

[20] J.-H. Seo, H. Sugiyama, and A. A. Shabana, "Three-dimensional large deformation analysis of the multibody pantograph/catenary systems," Nonlinear Dynamics, vol. 42, no. 2, pp. 199-215, 2005.

[21] J.-H. Lee and T.-W. Park, "Development of a three-dimensional catenary model using cable elements based on absolute nodal coordinate formulation," Journal of Mechanical Science and Technology, vol. 26, no. 12, pp. 3933-3941, 2012.

[22] I. Romero, "A comparison of finite elements nonlinear beams: the absolute nodal coordinate and geometrically exact formulations," Multibody System Dynamics, vol. 20, pp. 51-68, 2008. 
[23] J. Gerstmayr and A. A. Shabana, "Analysis of thin beams and cables using the absolute nodal co-ordinate formulation," Nonlinear Dynamics, vol. 45, no. 1-2, pp. 109$130,2006$.

[24] A. Goetz, Introduction to differential geometry. Addison Wesley Publishing Company, 1970.

[25] F. Chinesta, A. Leygue, F. Bordeu, J. Aguado, E. Cueto, D. González, I. Alfaro, A. Ammar, and A. Huerta, "PGD-based computational vademecum for efficient design, optimization and control," Archives of Computational Methods in Engineering, vol. 20, no. 1, pp. 31-59, 2013.

[26] F. Chinesta, R. Keunings, and A. Leygue, The proper generalized decomposition for advanced numerical simulations: a primer. Springer Science \& Business Media, 2013.

[27] F. Chinesta, A. Ammar, A. Leygue, and R. Keunings, "An overview of the proper generalized decomposition with applications in computational rheology," Journal of Non-Newtonian Fluid Mechanics, vol. 166, no. 11, pp. 578-592, 2011.

[28] E. Nadal, F. Chinesta, P. Díez, F. Fuenmayor, and F. Denia, "Real time parameter identification and solution reconstruction from experimental data using the proper generalized decomposition," Computer Methods in Applied Mechanics and Engineering, vol. 296, pp. 113-128, 2015.

[29] E. Nadal, A. Leygue, F. Chinesta, M. Beringhier, J. Ródenas, and F. Fuenmayor, "A separated representation of an error indicator for the mesh refinement process under the proper generalized decomposition framework," Computational Mechanics, vol. 55, no. 2, pp. 251-266, 2015. 